\title{
Autophagy-Mediated Clearance of Free Genomic DNA in the Cytoplasm Promoted the Growth and Survival of Breast Cancer Cells
}

\section{Mengfei Yao}

Peking University School of Basic Medical Sciences

\section{Yaqian Wu}

Peking University School of Basic Medical Sciences

\section{Yanan Cao}

Peking University School of Basic Medical Sciences

\section{Haijing Liu}

Peking University School of Basic Medical Sciences

\section{Ningning Ma}

Peking University School of Basic Medical Sciences

\section{Yijie Chai}

Peking University School of Basic Medical Sciences

\section{Shuang Zhang}

Peking University First Hospital

Hong Zhang

Peking University First Hospital

\section{Lin Nong}

Peking University First Hospital

\section{Li Liang}

Peking University First Hospital

\section{Bo Zhang ( $\nabla$ zhangbo@bjmu.edu.cn )}

Peking University Health Science Centre https://orcid.org/0000-0002-2605-8986

\section{Research article}

Keywords: Autophagy, cGAS, Cytoplasmic DNA, Micronuclei, Breast cancer

Posted Date: February 19th, 2021

DOI: https://doi.org/10.21203/rs.3.rs-227734/v1 
License: (c) (1) This work is licensed under a Creative Commons Attribution 4.0 International License. Read Full License 


\section{Abstract}

Background: The cGAS (GMP-AMP synthase)-triggered senescence-associated secretory phenotype (SASP) in promotion of cancer progression has been extensively documented. However, the role of cGASmediated DNA autophagy is little evaluated in breast cancer cells.

Methods: Immunofluorescence, senescence associated- $\beta$-galactosidase staining (SA- $\beta$-gal) and Western blot were performed to detect gene expression, distribution and phenotypes. PCR, IP-PCR, FISH, BrdU, Comet assay, coimmunoprecipitation, sucrose density gradient centrifugation were carried out to detect possible mechanisms. Trypan blue exclusion, Live/dead staining and MTS assay were to measure the cell viability. All analyses were performed using GraphPad Prism 8. Relationships were analyzed using t-tests. A P-value of less than 0.05 was considered significant. All statistical tests and $P$ values were 2-sided, and

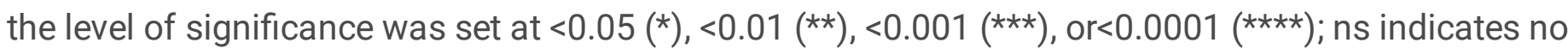
significance.

Results: Active DNA autophagy but not SASP activity could be detected in breast cancer cells with high micronucleus (MN). The selective autophagy of free genomic DNA in the cytoplasm is mediated by cGAS and usually coordinated with SQSTM1-mediated autophagy of ubiquitinated histones in breast cancer cell lines with high frequency of $\mathrm{MN}$ formation. Cytoplasmic DNA together with nuclear proteins derive from DNA replication-induced nuclear damage and $\mathrm{MN}$ collapse in breast cell lines which with severe DNA damage. The inhibition of DNA autophagy through either chemical inhibitors or genomic silencing of cGAS or SQSTM1 suppresses the growth and survival of breast cancer cells, while enhanced DNA damage increases the sensitivity to these inhibitors for more cancer cells. Human cancer cells with either high DNA autophagy or enhancement of DNA damage are sensitive to inhibition of DNA autophagy.

Conclusions: Our investigation revealed DNA autophagy in breast cancer cells with high MN formation. Autophagy of genomic DNA in the cytosol could be mediated by cGAS but is usually coordinated with other autophagic mediators. The selective autophagy mediated clearance of free genomic DNA protects of growth and survival in breast cancer cells even, and autophagic inhibition could be a potential therapeutic approach for cancer cells with high DNA autophagic activity.

\section{Background}

cGAS is an enzyme that catalyzes GTP and ATP to form cyclic dinucleic 2', $3^{\prime}$-cGAMP to stimulate stimulator of interferon gene (STING) and activate the kinases TBK1 and IKK, inducing the production of proinflammatory factor type I interferon [1]. cGAS-STING has been identified as an innate immune mechanism, but many recent studies have shown that CGAS-STING plays a major role in the activation of the senescence-associated secretory phenotype (SASP), in which senescent cells secrete many cytokines, growth factors, proteases and chemokines acting through either autocrine or paracrine mechanisms to promote inflammation [2-6]. The SASP is a crucial biological factor involved in aging-related diseases such as chronic inflammation, tissue degeneration or organ retardation. More importantly, the SASP has been revealed to be responsible for preventing the growth of cancer cells through its role as a tumor 
suppressor in the late stages of tumor progression via genomic instability of cancer cells and remodeling of the tumor microenvironment [7]. More interestingly, recent studies have also demonstrated that cGASSTING could induce autophagy upon binding to dsDNA through either the cGAS interaction with Beclin-1 or STING-mediated LC3 lipidation. Nevertheless, either SASP (proinflammatory) or autophagy is important in host innate defense [8]. However, cGAS-STING-mediated SASP or autophagy and autophagy have not been fully elucidated in cancer cells.

Activation of cGAS has been confirmed upon its binding to DNA, and in vitro analysis proved that DNA could effectively recruit cGAS through phase transition [9]. In living cells, cytoplasmic DNA, which can be exogenous, such as that from pathogenic organisms, and endogenous DNA of host cells, is a trigger that activates cGAS. Micronuclei (MNs) are small nuclei separated from the main nucleus. Similar to the main nucleus, MNs are encapsulated by the nuclear membranes and contain DNA and related substances [10]. MNs are prevalent in cancer cells and are believed to be a consequence of DNA damage and aberrations in mitosis $[11,12]$. Moreover, studies have suggested that the existence of cytosolic DNA is closely related to MNs [6]. MNs have been identified as the major source of cytoplasmic DNA involved in the activation of the cGAS-STING machinery to promote cancer progression and metastasis [13-15]. Moreover, free DNA derived from ecc rDNA (extrachromosomal circular rDNA) could also trigger cGAS-STING activity [16]. In addition, endogenous cytoplasmic DNA could be derived from mitochondria, which are injured under many circumstances [17]. Nevertheless, in contrast to its role in triggering the SASP phenotype by free DNA, cGAS-STING-mediated DNA-induced autophagy (DNA autophagy) in cancer cells has rarely been evaluated.

The mechanism by which the CGAS-STING pathway mediates either SASP or autophagy in response to exogenous pathogens could lead to their eradication. However, it is unclear how cGAS-STING makes decisions in response to endogenous DNA in cells. In the described investigation, we unexpectedly found that the BT-549 breast cancer cell line with a high frequency of MN formation presented a low SASP phenotype but high autophagic activity, and subsequent experiments showed that its high DNA autophagy mediated by cGAS and cytosol-free DNA was closely related to MN formation and DNA damage, and inhibition of DNA autophagy could suppress its growth and survival. Furthermore, expanded observations indicated that enhancement of DNA damage or cancer cells with high relative DNA autophagy could increase DNA autophagy and sensitize the cells to autophagic inhibitors.

\section{Materials And Methods}

\section{Reagents and antibodies}

The anti-Lamin B1 rabbit polyclonal antibody, anti-Beclin1 rabbit polyclonal antibody, anti-STING (TMEM173, EPR13130) rabbit monoclonal antibody, and anti-DNase II rabbit monoclonal antibody were purchased from Abcam (Cambridge, UK). The Stat6 (D-1) mouse monoclonal antibody, Lamin B1 mouse monoclonal antibody and cGAS (D-9) were purchased from Santa Cruz Biotechnology, Inc. (CA, 95060, USA), and anti-phospho-histone $\mathrm{yH} 2 \mathrm{AX}$ mouse monoclonal antibody (Ser139) and anti-RPA2 mouse 
monoclonal antibody were from Millipore (Billerica, MA, USA). The anti-Lamin A/C (R386) rabbit polyclonal antibody, anti-IRF3 rabbit polyclonal antibody and anti-LAMP2 rabbit polyclonal antibody were from Bioworld Technology, Inc. (MN, USA). Anti-SQSTM1 rabbit polyclonal antibody and anti-LC3 rabbit polyclonal antibody were purchased from MBL, Ltd. (Chiba, Japan). Anti-phospho-STING (Ser366) rabbit monoclonal antibody and phospho-IRF3 rabbit monoclonal antibody were purchased from Cell Signaling Technology (Danvers, MA, USA). Nuclear Fast Red Staining Solution (0.1\%; G1320), LysoTracker and DAPI (C0060) were purchased from Solarbio (Beijing, China). The full-length expression plasmids Flag-cGAS, Flag-SQSTM1 and Flag-Beclin-1 were purchased from YouBio, Inc. (Beijing, China). Bafilomycin A1 and H151 were purchased from Selleck (Shanghai, China), and chloroquine (CQ) and 2,3'-cGAMP were purchased from Sigma Aldrich (St. Louis, MO, USA).

\section{Cell culture and treatment}

Breast cancer cells: MDA-231, MCF-7, BT-549, and other cancer cells: 786-0, DU145, PC-3M, HCT-116, and HeLa were maintained in 1640 or Dulbecco's modified Eagle's medium with high glucose (Gibco, Life Technologies, Grand Island, NY, USA) supplemented with $10 \%$ fetal bovine serum. The cells were incubated in a humidified atmosphere with $5 \% \mathrm{CO}_{2}$ at $37^{\circ} \mathrm{C}$.

\section{Small interfering RNA}

RNAi was designed and synthesized by GenePharma (Suzhou, China). RNAi was performed by the transfection of siRNA oligos using Lipofectamine 2000 transfection reagent (Invitrogen) according to the manufacturer's instructions. The sequences are as follows: si-cGAS-1: Forward: 5'-

GGCCUCUGCUUUGAUAACUTT-3', Reverse: 5'-AGUUAUCAAAGCAGAGGCCTT-3'; si-cGAS-2: Forward: 5'GGCUAUCCUUCUCUCACAUTT-3'; Reverse: 5' -AUGUGAGAGAAG GAUAGCCTT-3'. si-LC3-1: Forward: 5'GCUACAAGGGUGAGAAGCATT-3'; Reverse: 5'-UGCUUCUCACCCUUGUAGCTT-3'; si-LC3-2: Forward: 5'GCGAGUUGGUCAAGAUCAU TT-3'; Reverse: 5'-AUGAUCUUGACCAACUCGCTT-3'; si-LC3-3: Forward: 5' GCUUCCUC UAUAUGGUCUATT-3'; Reverse: 5'-UAGACCAUAUAGAGGAAGCTT-3'. si-SQSTM1-1: Forward: 5'AGAUUCGCCGCUUCAGCUUTT-3'; Reverse: 5'-AAGCUGAAGCGGCGAAUC UTT-3'; si-SQSTM1-2: Forward: 5'CGCUCACCGUGAAGGCCUATT -3'; Reverse: 5'-UAGGC CUUCACGGUGAGCGTT-3'; si-SQSTM1-3: Forward: 5'GCACUACCGCGAU GAGGACTT-3'; Reverse: 5'-GUCCUCAUCGCGGUAGUGCTT-3'『si-DNase2-1: Forward: 5'GGCAGCCU GUAGACUGGUUTT-3'; Reverse: 5' -AACCAGUCUACAGGCUGCCTT-3'; si-DNase2-2: Forward: 5'GCAUACAGCUGGCCUCAUATT-3'; Reverse: 5'-UAUGAGGCCAGCUGUAUG CTT-3'. The control RNAi (siNC) was composed of scrambled sequences.

\section{Western blotting}

Total cell lysates were obtained by incubating the cells in $2 \times$ SDS for 30 minutes at $4^{\circ} \mathrm{C}$. After centrifugation at $10,000 \times \mathrm{g}$ for 10 minutes at $4^{\circ} \mathrm{C}$, the supernatant was collected and stored at $-20^{\circ} \mathrm{C}$ for 
subsequent analysis. For cell fractions, cytoplasmic and nuclear proteins were extracted using nuclear and cytoplasmic protein extraction kits (Sangon Biotech Co., Shanghai, China), respectively. Equal amounts of cell proteins (20-40 $\mu \mathrm{g} / \mathrm{lane}$ ) were separated by SDS-PAGE in $10 \%$ gels and transferred to PVDF membranes (Millipore, Billerica, MA, USA) using a semidry transfer cell (Bio-Rad,Hercules, CA, USA) at $25 \mathrm{~V}$ for 60 minutes. The membranes were then blocked for 1 hour with TBS-T $(20 \mathrm{mM}$ Tris-HCl pH 7.6, $137 \mathrm{mM}$ $\mathrm{NaCl}$ and $0.1 \%$ Tween-20) containing 5\% nonfat dry milk (Cell Signaling Technology, Beverly, MA, USA) or with $1 \%$ BSA (Sigma Aldrich) and incubated overnight with primary antibodies. After the membranes were washed, they were incubated for 1 hour with peroxidase-conjugated goat anti-rabbit IgG or peroxidaseconjugated goat anti-mouse IgG. The proteins were visualized using an enhanced chemiluminescence kit (Bio-Rad, CA, USA). Band images of three independent experiments were quantified by optical density using Lab-Works 4.6 software (Bio-Rad, CA, USA). $\beta$-actin was used as an internal control for each protein. The antibodies included anti-LC3 (1:1000), anti- $\beta$-actin (1:1000), anti-cGAS (D-9) (1:500), anti-SQSTM1 (1:1000), anti-DNase2 (1:1000), anti-lamin A/C (R386) (1:500), anti-IRF3 (1:500), anti-TMEM173 (1:1000), and anti-LAMP2 (1:1000).

\section{Immunofluorescence}

Immunofluorescence staining was performed as described previously [18]. The results were observed and recorded using a fluorescence microscope (Model CX51; Olympus, Tokyo, Japan), and Photoshop version 7.0 (Adobe Systems, Inc.) was used to analyze the results. The antibodies used included anti-LC3 (1:1000), anti- $\beta$-actin (1:1000), anti-cGAS (D-9) (1:500), anti-SQSTM1 (1:1000), anti-DNase II (1:1000), anti-lamin A/C (R386) (1:500), IRF3 (1:500), anti-STING (1:1000), and anti-LAMP2 (1:1000).

\section{Senescence associated- $\beta$-galactosidase staining (SA- $\beta$-gal)}

Cells were subjected to SA- $\beta$-gal staining using an SA- $\beta$-gal staining kit (GenMed, GenMed Scientifics, Inc., USA) according to the manufacturer's directions. One hundred cells were counted in random fields, and the percentage of SA- $\beta$-gal-positive cells was calculated. Cell counting was repeated three times, and the mean was calculated.

\section{Electron microscopy}

Cells were fixed with $0.1 \mathrm{M}$ sodium phosphate buffer ( $4 \%$ paraformaldehyde and $0.1 \%$ glutaraldehyde) at $4^{\circ} \mathrm{C}$ overnight. The samples were embedded with Lowicryl K4M (BioChemika), and sections were prepared with the Leica EM UC7 ultramicrotome and then stained with saturated uranyl acetate. The sections were observed under a transmission electron microscope (JEOL-1230, Japan) and recorded.

\section{Co-immunoprecipitation}


A total of $1 \times 10^{7}$ transfected cells were washed twice with PBS, $600 \mu$ l of precooled hypo-osmic buffer (25 $\mathrm{mM}$ Tris, $\mathrm{pH} 7.4,85 \mathrm{mM} \mathrm{KCl}$ ) was added, and the samples were incubated on ice for 30 minutes and centrifuged at $4^{\circ} \mathrm{C}$ at $12000 \mathrm{rpm}$ for 10 minutes. The supernatant was saved and incubated with Flag antibody-conjugated agarose beads (MBL, Chiba, Japan) and gently shaken on a turntable overnight at $4^{\circ} \mathrm{C}$. The beads were washed with hypo-osmic buffer containing protease inhibitor cocktail for 10 minutes; this process was repeated 4 times. Finally, the beads were dissolved in 1.5xSDS loading buffer, and $30 \mu l$ of supernatant was analyzed by Western blotting. The primary whole cytoplasmic supernatant was used as input.

\section{IP-PCR}

A total of $2 \times 10^{7}$ transfected cells were washed with preheated PBS at $37^{\circ} \mathrm{C} 3$ times, fixed with $1 \%$ formaldehyde in PBS in a $37^{\circ} \mathrm{C}$ incubator for 15 minutes, quickly washed with ice-cold PBS 5 times, scraped into an Ep tube, and centrifuged at $800 \mathrm{~g}$ at $4^{\circ} \mathrm{C}$ for 3 minutes. Then, the supernatant was discarded, and $500 \mu$ l of hypo-osmic buffer ( $25 \mathrm{mM}$ Tris, pH 7.4, $85 \mathrm{mM} \mathrm{KCl}$ ) was added to isolate the cytoplasmic protein. Part of the supernatant was saved as the input. The remaining part was used for IP experiments. An appropriate amount of $500 \mu$ l elution buffer ( $1 \%$ SDS, $0.1 \mathrm{M}$ sodium bicarbonate was used to elute the protein-DNA complex on the beads for 10 minutes at room temperature. Then, RNase $A$ was added, the samples were heated at $37^{\circ} \mathrm{C}$ and shaken for $2 \mathrm{~h}$, Proteinase $\mathrm{K}$ was added, and the samples were heated at $55^{\circ} \mathrm{C}$ and shaken for $2 \sim 3 \mathrm{~h}$. Then, the samples were heated at $65^{\circ} \mathrm{C}$ and shaken overnight to isolate the protein-DNA complexes. Finally, the IP DNA was extracted by the phenol and chloroform method, and the results were analyzed by PCR.

\section{Sucrose density gradient centrifugation}

Gradient concentrations of sucrose solution ( $5 \%-40 \%$, the concentration interval was $5 \%$ ) with protease inhibitor cocktail were established as described [19]. The cytoplasmic proteins, extracted by hypo-osmic buffer as described above, were carefully dropped on the top layer and centrifuged at 40,000 rpm (Beckman, Brea, CA, USA) for 4 hours at $4^{\circ} \mathrm{C}$. After centrifugation, the samples were carefully collected from a $500 \mu$ l aliquot of each fraction, and the aliquot of each fraction was analyzed by Western blot. Cytoplasmic DNA was extracted from $100 \mu$ l of each fraction and analyzed by PCR.

\section{Acid extraction of cytoplasmic histones}

Cytoplasmic histones were isolated by acid extraction methods with some modifications [20]. Briefly, cytoplasmic proteins from $1 \times 10^{7}$ cells of each kind, extracted by hypo-osmic buffer as described above, were slowly added to $0.4 \mathrm{~N} \mathrm{H}_{2} \mathrm{SO}_{4}(500 \mu \mathrm{l}$ of $\mathrm{H} 2 \mathrm{SO} 4$ to a $100 \mu \mathrm{l}$ cytoplasmic solution) and incubated at $4^{\circ} \mathrm{C}$ with intermittent rotation for 2 hours. After centrifugation at $5500 \mathrm{rpm}$ for 5 minutes, the supernatants were gently added to $150 \mu$ l of $100 \%$ TCA (final concentration of $20 \%$ ) and kept on ice for at least 5 hours 
without agitation. After centrifugation, the pellets were washed with $500 \mu \mathrm{l}$ of acetone $+0.1 \% \mathrm{HCl}$, and the tubes were left open overnight to evaporate the acetone. The pelleted histones were resuspended in $20 \mu l$ of $\mathrm{ddH}_{2} \mathrm{O}$ and subsequently analyzed by SDS-PAGE and Western blotting.

\section{PCR detection}

DNA in nuclear or cytoplasmic were separately extracted. The cells were cultured in $10 \mathrm{~cm}$ dishes and counted. A total of $1 \times 10^{3}$ cells were treated with hypo-osmic buffer ( $25 \mathrm{mM}$ Tris pH 7.4, $\left.85 \mathrm{mM} \mathrm{KCl}\right)$ and centrifuged, and the supernatant (the cytoplasmic components) was saved. Then, the cytoplasmic DNA was extracted by the phenol and chloroform method. The sediment (nuclear DNA) was extracted by Tigen Kits (Beijing, China). PCR was performed with using Alu and rDNA primers: Alu: Forward: 5'CCTGAGGTCAGGAGTTCGAG-3'; Reverse: 5'-CCCGA GTAGCTGGGATTACA-3' (115 bp); 5.8S rDNA: Forward: 5'- GAGGCAACCCC CTCTCCTC TT-3'; Reverse: 5'-GAGCCGAGTTCACCGCTA-3' (136 bp); 18S rDNA: Forward: 5'-CGCCG CGCTCTACCTTACCTA-3'; Reverse: 5' -TAGGAGAGGAGCGAGCGACCA-3' (159 bp); $28 \mathrm{~S}$ rDNA: Forward: 5' -CTCCGAGACGCGACCTCAGAT-3'; Reverse: 5'-CGGGTCTT CCGTAC GCCACAT-3' (173 bp). Quantitative real-time RT-PCR was performed on a PCR system (Applied Biosystems, Inc., Carlsbad, CA, USA) using SYBR Premix. The results were evaluated as the ratio of cytoplasmic: nuclear DNA; cytoplasmic DNA was normalized to nuclear DNA. The results were analyzed by GraphPad Prism 8.0.

\section{FISH}

The Alu probes were as follows: Alu-1 :5'-CCTGAGGTCAGGAGTTCGAGACCAGCCT-3'; Alu-2: 5'ACGCCTGTAATCCCAGCACTTTGGGAGG-3'; Alu-3:5' -TCGCGCCACTGCACTC CAGCCTGGGCGA-3'. They were synthesized and conjugated with a single Quasar 570 molecule at the 5 ' end (Sango Biotech, Shanghai, China). Cells grown on cover slides were fixed in $3 \%$ paraformaldehyde ( $\mathrm{pH} 7.4$ ) containing $0.1 \%$ Triton-X 100 for 30 minutes and permeabilized in $0.1 \%$ Triton $X-100$ for 20 minutes. After denaturation at $95^{\circ} \mathrm{C}$ for 5 minutes, hybridization was performed in a mixture of probes ( $20 \mathrm{ng} / \mathrm{per}$ slide) and $35 \%$ deionized formamide, $10 \%$ dextran sulfate, $1 \times$ Dehart's, and $2 \times S S C$ for 20 hours at $42^{\circ} \mathrm{C}$. The slides were washed for 40 minutes in $2 \times$ SSC with several changes. Nuclei were stained with Hoechst 34580 (Sigma Aldrich) for 10 minutes, and the results were observed and recorded using a fluorescence microscope (Model CX51, Olympus, Tokyo, Japan), and Photoshop version 7.0 (Adobe Systems, Inc. San Jose, CA, USA) was used to observe and analyze the results. At least 500 cells were evaluated, and the results were evaluated as the ratio of the intensity in cytoplasmic and nuclear DNA; cytoplasmic signals were normalized to those of the nuclei.

\section{Comet assay}

The comet assay was performed using a comet assay kit according to the manufacturer's instructions. First, $1 \times 10^{5}$ cells were prepared. Neutral or alkaline electrophoresis was performed. The slides were viewed 
by epifluorescence microscopy using a FITC filter. The results were analyzed by Comet Score software (Version 2.0038).

The comet assay was performed using a comet assay kit (Abcam, Cambridge, UK) according to the manufacturer's instructions. First, $1 \times 10^{5}$ cells were prepared. Comet Agarose was pipetted onto the Comet Slide to form base layer cells, which were combined with Comet Agarose at $37^{\circ} \mathrm{C}$. Cell samples were combined with Comet Agarose at a 1/10 ratio (v/v). We pipetted the agarose/cell mixture on top of the base layer. The cells were treated with lysis buffer and alkaline solution. Electrophoresis was performed under alkaline or neutral conditions. Voltage was applied to the chamber for $10-15$ minutes at $1 \mathrm{volt} / \mathrm{cm}$. The cells were stained with DNA dye. The slides were viewed by epifluorescence microscopy using a FITC filter. The results were analyzed by Comet Score software (Version 2.0038).

\section{BrdU incorporation assay}

BrdU incorporation assays were performed as described previously (18). Briefly, BrdU (10 $\mu \mathrm{M})$ was added to the culture medium for 30 minutes before analysis, and then, the cells were fixed with $4 \%$ formaldehyde, permeabilized with $0.1 \%$ Triton X-100, and denatured with $20 \mathrm{mM} \mathrm{HCl}$ in $150 \mathrm{mM} \mathrm{NaCl}$ and $3 \mathrm{mM} \mathrm{KCl}$ for 20 minutes at $25^{\circ} \mathrm{C}$. The cells were then incubated with a primary antibody mixture composed of primary antibodies (BrdU and MCM7 or Lamin B1).

\section{Trypan blue exclusion assay}

Cell growth was determined by Trypan blue exclusion assays with a Trypan Blue Staining Cell Viability Assay Kit (Beyotime, Shanghai, China). Cells $\left(1 \times 10^{4}\right.$ cells/per well) grown with or without treatment with CQ, bafilomycin A1, or H-151 in 96-well plates were harvested, and $50 \mu$ l of trypan blue was added to a $50 \mu \mathrm{l}$ cell suspension according to the manufacturer's protocol. Viable cells were counted under a microscope with a hemocytometer. The assays were performed in triplicate and repeated at least three times.

\section{Live/dead viability assay}

A live/dead assay was performed using a live/dead cell viability assay kit (Abcam, Cambridge, UK). A total of $1 \times 10^{5}$ cells were seeded in 12-well or 96 -well plates and incubated for 24 hours. The cells were treated with CQ or bafilomycin A1 and incubated for the indicated times. Subsequently, the cells were rinsed twice with PBS before the fluorochromes were added and incubated for $\mathbf{4 5}$ minutes. Fluorescence images were then taken (Model CX51, Olympus, Tokyo, Japan), and live or dead cells were counted and calculated.

\section{MTS assay}


MTS assays were performed using an MTS assay kit (Bestbio, Shanghai, China). A total of $1 \times 104$ cells were seeded in 96-well plates and incubated for 24 hours. The cells were treated with CQ or bafilomycin A1 and incubated for the indicated times. Ten microliters of MTS solution were added to each well and incubated for 3 hours at $37^{\circ} \mathrm{C}$. The absorbance was measured at $490 \mathrm{~nm}$, and cell viability was analyzed.

\section{Statistical analysis}

All analyses were performed using GraphPad Prism 8 (CA, USA) and Excel 2010 (WA, USA). Relationships were analyzed using t-tests. A P-value of less than 0.05 was considered significant. All statistical tests and $P$ values were 2-sided, and the level of significance was set at $<0.05(*),<0.01(* *),<0.001(* * *)$, or $<0.0001$ (***); ns indicates no significance.

\section{Results}

\section{A high frequency of MNs in breast cancer cells resulted in an autophagic phenotype}

MCF-7, MDA-231, and BT-549 breast cancer cells were subjected to immunofluorescence staining for Lamin B1 and NAT10 as described previously[18], and spontaneous MN formation in each kind of cell was analyzed. The rate of spontaneous MNs in the MCF-7 cells was approximately $5 \%$, that in the MDA-231 cells was approximately $15 \%$, and that in the BT-549 cells was approximately $35 \%$ (Fig. 1a). However, the MNs formed in three cell lines generally contained DNA and Lamin B1 or A/C, nuclear lamina proteins, and other nuclear envelope components, including LBR (Lamin B receptor), nuclear pore complex components (MAB414, nucleoporin 153), the nuclear basket protein TPR (translocated promoter region), and integral membrane components (Sun2 and nesprin2), suggesting that the nuclear membrane of MNs generally maintained structural components similar to the membrane of the main nuclei (Supplementary Fig. S1a).

MNs are considered a major source of free DNA, which triggers the activation of the SASP through DNA binding to CGAS-STING pathway components [6]. Therefore, the MDA-231, BT-549, and MCF-7 cells were analyzed by SA- $\beta$-gal staining, and the results indicated that the MCF-7, MDA-231 cells showed $4 \%$ and $8 \%$ SA- $\beta$-gal positivity, while the BT-549 cells unexpectedly showed no SA- $\beta$-gal-positive cells even with repeated staining (Fig. 1b).

Subsequent Western blotting analysis showed the expression of the STING pSTING, IRF3, STAT6 in BT-549 cells is lower than that of MDA-231 or MCF-7 cells, indicating that the activity of SASP in BT-549 cell line was significantly lower than that in MDA-231 and MCF-7 cells (Fig. 1C). Staining also showed that after treatment with the STING antagonist H-151 $(2 \mu \mathrm{M})$ for 24 hours, the positive ratio strongly decreased, confirming that the SASP phenotype is mediated via cGAS-STING in the MDA-231 cells (Supplementary Fig. S1b). However, treatment with the STING agonist cGAMP [1] and transfection with Flag-cGAS or FlagSTING did not induce SA- $\beta$-gal staining, indicating blockade of CGAS-STING signaling in the BT-549 cells (data not shown). 
Recently, the cGAS-STING pathway was shown to mediate DNA autophagy. Therefore, autophagic activity was first compared among the MCF-7, MDA-231 and BT-549 cells. Western blotting showed that the BT549 cells presented high expression of LC3, SQSTM1, and LAMP2, which was not obvious in the MDA-MB231 or MCF-7 cells, and notably, a high level of DNase II was detected in the BT-549 cells (Fig. 1d). The BT549 cells were treated with the autophagic inhibitors CQ $(10 \mu \mathrm{M})$ for 4 hours or bafilomycin $\mathrm{A} 1(10 \mathrm{nM})$ for 24 hours, and the levels of LC3, SQSTM1 and LAMP2 were strongly increased, confirming the autophagic activity in the BT-549 cells (Fig. 1e). Moreover, the expression of DNase II was dose-dependently enhanced by CQ treatment $(10 \mu \mathrm{M})$ in the BT-549 cells (Fig. 1e). Furthermore, LysoTracker staining showed that lysosomes were more abundant in the BT-549 cells than in the MDA-231 cells (Fig. 1e). The results suggested the involvement of DNA autophagy in the BT-549 cells.

To further confirm the autophagic activity, we also performed electron microscopy of the MDA-MB-231 and BT-549 cells after treatment with bafilomycin A1 (10 nM) for 72 hours, and the results showed that there were many autophagic vesicles in the BT-549 cells but not the MDA-MB-231 cells (Fig. 1g).

Therefore, the above results demonstrated that breast cancer cells with MNs could differentially present SASP and autophagy, and cells with a high frequency of MNs presented an autophagic phenotype.

\section{Detection of genomic DNA autophagy in the cytoplasm of breast cancer cells}

High autophagic activity and frequent MN formation, as well as DNase II expression, indicated the possibility of active DNA autophagy in the BT-549 cells. First, SQSTM1 and LC3, the core proteins of autophagy, were stained by immunofluorescence in the MCF-7, MDA-231 and BT-549 cells. The results showed that the BT-549 cells presented marked cytoplasmic SQSTM1-positive granules or vesicles ( $60 \%$ cells) (Fig. 2a), but only a few cells showed cytoplasmic staining for SQSTM1 in MCF-7 and MDA-231. All three kinds of cells presented SQSTM1 positive in occasional Lamin B1-outlined MNs ( 5\% MNs) (Supplementary Fig. S2a). Similarly, the BT-549 cells also presented many cytoplasmic LC3-positive vesicles and fewer LC3-positive vesicles than the MDA-231 or MCF cells (Fig. 2a, b). However, almost no LC3 was detected in the MNs of the three kinds of cells (Supplementary Fig. S2b).

Interestingly, the distribution of CGAS was shown to be similar to that of SQSTM1 or LC3. The BT-549 cells presented accumulation of cytoplasmic cGAS granules that colocalized with SQSTM1 ( 50\%) and cytoplasmic LC3 ( 40\% cells), while the MCF-7 and MDA-231 cells were faintly stained for CGAS (Fig. 2a, b). All three kinds of cells showed staining for cGAS in occasional MNs (3\%-5\%) (Supplementary Fig. S2c). In addition, only a few cGAS-positive MNs ( 3\%) were positive for SQSTM1 but not LC3 (Fig. 2b, 2c). STING staining showed that a few cytoplasmic puncta were detected in the MCF-7 and MDA-231 cells but were distributed in the Golgi apparatus in the BT-549 cells ( 30\%), while the staining was generally weak (see Supplementary Fig. S3c).

Further, Beclin-1, another autophagy mediator, was stained in breast cancer cells, but few cytoplasmic positive vesicles were detected and only occasional staining in cGAS positive MN (Supplementary Fig. 
S2d, S2e).

To further confirm the autophagic activity, we treated the MDA-MB-231 and BT-549 cells with CQ $(10 \mu \mathrm{M})$ or bafilomycin A1 (10 nM) for 24 hours, and the results showed that the BT-549 cells presented marked increases in cytoplasmic cGAS, SQSTM1 and LC3, while the MDA-231 and MCF-7 cells only showed slight increases in a few cells (Fig. 2a, b). However, the MNs in the three kinds of cells presented no significant increase in positive staining of CGAS, SQSTM1, LC3 or Beclin-1 (data not shown). As expected, treatment with bafilomycin A1 markedly increased not only the levels of SQSTM1, LC3, and LAMP2 but also cGAS and STING in the BT-549 cells (Fig. 2d).

The colocalization and accumulation of SQSTM1, CGAS and LC3 in the cytoplasm suggested the possibility of selective cytoplasmic DNA autophagy. To directly verify the genomic DNA in the cytoplasm, we independently isolated cytoplasmic DNA from the MCF-7, MDA-231 and BT-549 cells and the existence of genomic DNA was determined by PCR detection of Alu- or rDNA (5.8S, $18 \mathrm{~S}$ and $28 \mathrm{~S}$ ) repeated sequences. The gel electrophoresis results showed that the cytoplasmic abundance of Alu in the BT-549 cells was significantly higher than that in the MDA-231 and MCF-7 cells (Fig. 2e). Moreover, real-time PCR confirmed the greater cytoplasmic abundance of Alu and rDNA (5.8S, $18 \mathrm{~S}$ and $28 \mathrm{~S})$ in the BT-549 cells than in the MCF-7 and MDA-231 cells (Fig. 2e).

Given that free cytosolic DNA was not membrane enclosed, coimmunoprecipitation was carried out to explore the potential interaction between CGAS or SQSTM1 and genomic DNA in the cytoplasm. FlagSQSTM1 and Flag-cGAS were transfected into the MDA-231 and BT-549 cells, and the cytoplasmic fractions of Flag-SQSTM1 and Flag-cGAS were immunoprecipitated. The sequences of Alu- or 5.8S rDNA could be detected in either precipitated Flag-SQSTM1 or Flag-cGAS and were more abundantly detected in the BT-549 cells than in the MDA-231 cells (Fig. 2f). Moreover, the sequences of Alu DNA in the cytoplasm showed greater detection in the BT-549 cells than in the MDA-231 cells by FISH, and following treatment with CQ and bafilomycin A1, the cytoplasmic signals were enhanced in the BT-549 cells (Supplementary Fig. S2f).

Taken together, these data indicated that DNA autophagy in breast cancer cells could be selective autophagy of cytoplasmic free DNA but not nucleophagy and possibly involved cGAS, SQSTM1 and LC3.

\section{DNA autophagy in the cytoplasm was involved in the coordination of CGAS and SQSTM1}

To explore the autophagic flow in DNA autophagy, we investigated the relationship between cGAS and SQSTM1 or LC3. After knockdown of either LC3 or SQSTM1 by siRNA, the level of cGAS was obviously increased in the BT-549 cells, as shown by Western blotting (Fig. 3a, b), while the granular form of cGAS was reduced, as shown by immunofluorescence staining (Fig. 3a, b). Moreover, depletion of LC3 increased the SQSTM1 levels (Fig. 3a). In contrast, after knockdown of $c G A S$ by siRNA, the levels of either LC3 or SQSTM1 were decreased in the BT-549 cells, as shown by immunofluorescence and Western blotting (Fig. 3c). 
After knockdown of cGAS by interfering RNAs, gel electrophoresis showed that the levels of cytoplasmic Alu- and rDNA sequences increased obviously in the BT-549 cell lines but not in the MCF-7 or MDA-231 cell lines (Fig. 3d). Similarly, knockdown of LC3 also resulted in the same findings (Fig. 3d).

To explore the potential autophagic complex of SQSTM1, we further analyzed CGAS, LC3 and free DNA through cytoplasmic fractioning in a density gradient fraction assay, in which the detection of SQSTM1 overlapped in fractions containing cGAS, LC3 and free DNA (Fig. 3e). Moreover, the potential complex of cGAS, SQSTM1 and genomic DNA was further analyzed by a coprecipitation strategy, which showed that genomic DNA could be detected in the MDA-231 and BT-549 cells transfected with either Flag-SQSTM1 or Flag-cGAS (Fig. 2f). In addition, endogenous CGAS in the BT-549 cells coprecipitated with transfected FlagSQSTM1 (Supplementary Fig. S3a).

However, there is no direct interaction between SQSTM1 and cGAS or DNA binding activity, and how SQSTM1 participates in DNA autophagy needs to be addressed. Generally, SQSTM1 recognizes ubiquitinated substances during autophagy, and it has been reported that cGAS undergoes K48-linked ubiquitination at K414, leading to SQSTM1-dependent selective autophagic degradation [21]. Thus, FlagSQSTM1 was transfected into the BT-549 cells, but K48-ubiquitinated cGAS could not be detected by either Western blotting or immunofluorescence staining (data not shown).

Since DNA is usually coated with histones or other chromatin-binding proteins, cytoplasmic free DNA was also assumed to be bound to histones. To clarify this, we isolated cytoplasmic histones from the BT-549 or MDA-231 cells by acid-based extraction [20]. Western blotting showed that cytoplasmic histones could be detected in both the BT-549 and MDA-231 cells, but the BT-549 cells presented more cytoplasmic histones than the MDA-231 cells (Fig. 3f); these structures could be detected by anti-K48 ubiquitin and FK2 antibodies (against poly- or monoubiquitinated proteins) (Fig. 3f). Moreover, immunofluorescence staining showed that more intensive staining of FK2 could be detected in the cytoplasm of the BT-549 cells than in that of the MDA-231 cells (Supplementary Fig. S3b).

Recent reports suggest that activation of CGAS upon binding to DNA could trigger activation of STING, leading to either SASP or autophagy, and MCF-7, MDA-231 and BT-549 cells presented different endogenous levels of STING or phosphorylated STING, which was consistent with their SASP and autophagic phenotypes (Fig. 1b, c). Moreover, endogenous or exogenous STING was present in a few cytoplasmic vesicles in the MCF-7 and MDA-231 cells but was distributed in the Golgi apparatus in the BT549 cells and could be disrupted by brefeldin A (BFA) (Supplementary Fig. S3c). In the BT-549 cells, the level of STING markedly increased in the presence of bafilomycin A1 (10 nM) (Fig. 2d) or with downregulation of DNase II (Supplementary Fig. S3d). However, after treatment of the BT-549 cells with either the STING antagonist H-151 (2 $\mu \mathrm{M})$ or agonist CGAMP (300 nM) for 24 hours, SQSTM1 or LC3 presented no change in immunofluorescence staining (data not shown). Thus, degradation of STING may be involved in the autophagic process, while its activity in the regulation of autophagy in BT-549 cells requires further exploration (see Discussion). 
Taken together, the results suggested that free cytoplasmic DNA autophagy could be mediated in a complicated process involving cGAS binding to DNA and recognition of ubiquitinated histones by SQSTM1, consequently resulting in activation of LC3.

\section{Genomic DNA in the cytoplasm could be derived from either damaged nuclei or MNs}

Since the cytoplasmic DNA undergoing autophagy was genomic DNA from the nuclei and nuclear membrane in the breast cancer cells were not generally broken (Supplementary Fig. S1a), DNA damage was assumed to be involved. The level of DNA damage in the BT-549, MCF-7 and MDA-231 cells was analyzed by comet assays. Table 1 shows that the tail length of the BT-549 cells was substantially longer than that of the MDA-231 $(76.83, \mathrm{P}<0.0001)$ and MCF-7 cells $(70.59, \mathrm{P}<0.0001)$, the tail intensity of the BT-549 cells was greater than that of the MDA-231 (70319, $P<0.01)$, and MCF-7 cells $(75513, P<0.0001)$, and the tail movement of the BT-549 cells was also obviously higher than that of the MDA-231 (13.09) and MCF-7 cells $(15.66, P<0.0001)$. These results indicated that the DNA damage was the most severe in the BT-549 cells (Table 1, Fig. 4a). 
Table 1

The results of the Comet assay

\begin{tabular}{|c|c|c|c|c|c|c|c|}
\hline \multirow{2}{*}{$\begin{array}{l}\text { Cell } \\
\text { lines }\end{array}$} & & \multicolumn{2}{|l|}{ Tail length } & \multicolumn{2}{|l|}{ Tail intensity } & \multicolumn{2}{|c|}{ Tail movement } \\
\hline & & Mean & SD & Mean & SD & Mean & SD \\
\hline \multirow{5}{*}{$\begin{array}{l}\text { MDA- } \\
231\end{array}$} & Control & 76.83 & 45.00 & 70319.47 & 59133.3 & 13.09 & 14.16 \\
\hline & $\begin{array}{l}\text { Bafilomycin } \\
\text { A1 }\end{array}$ & 87.43 & 43.28 & 69149.58 & 61988.6 & 16.14 & 20.33 \\
\hline & NC & 78.88 & 46.56 & 69820.57 & 59621.2 & 12.78 & 13.21 \\
\hline & si-LC3 & 66.26 & 37.16 & 94829.15 & 116894 & 19.06 & 28.14 \\
\hline & si-cGAS & 74.13 & 61.62 & 72505.77 & 72552.6 & 13.19 & 11.86 \\
\hline \multirow{5}{*}{$\begin{array}{l}\text { BT- } \\
549\end{array}$} & Control & $109.94(* * * *)$ & 39.37 & 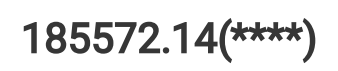 & 139055 & $42.63(* \star \star *)$ & 25.33 \\
\hline & $\begin{array}{l}\text { Bafilomycin } \\
\text { A1 }\end{array}$ & $182.468(* \star \star \star)$ & 71.71 & $321391.656(\star \star \star)$ & 183954 & $60.123(*)$ & 51.03 \\
\hline & NC & 100.67 & 45.72 & 189102.12 & 139234 & 45.22 & 25.43 \\
\hline & si-LC3 & $190.637(* \star \star *)$ & 81.25 & 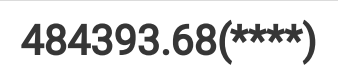 & 136164 & $95.130(* *)$ & 35.25 \\
\hline & si-cGAS & $163.324(* * * *)$ & 68.48 & $392065.161(* \star *)$ & 111780 & $70.662(* *)$ & 47.03 \\
\hline \multirow{5}{*}{$\begin{array}{l}\text { MCF- } \\
7\end{array}$} & Control & 70.59 & 41.03 & 75513.63 & 102705 & 15.66 & 21.45 \\
\hline & $\begin{array}{l}\text { Bafilomycin } \\
\text { A1 }\end{array}$ & 69.48 & 38.46 & 84516.05 & 114563 & 15.93 & 20.43 \\
\hline & NC & 68.23 & 40.92 & 79333.41 & 99012.3 & 16.01 & 21.22 \\
\hline & si-LC3 & 59.94 & 30.88 & $95231.169\left(^{*}\right)$ & 100438 & 15.09 & 21.39 \\
\hline & si-cGAS & 77.88 & 55.12 & 87188.13 & 93316.8 & 15.54 & 21.37 \\
\hline
\end{tabular}

Following bafilomycin A1 treatment, the length, intensity and movement of the tails in the BT-549 cells, but not in the MCF-7 and MDA-231 cells, increased significantly (182.47 ( $P=0.0008), 321391.66(P=0.0002)$, and 60.12 ( $P=0.0024)$, respectively) (Table 1, Fig. 4a). Similarly, knocking down cGAS and LC3 in BT-549 cells by siRNA also increased the tail values in the comet assay (Table 1, $\mathrm{P}<0.001$ ) (Table 1, Fig. 4a). These results indicated that inhibition of autophagy could influence the status of DNA damage.

Moreover, TUNEL assays were performed to directly measure DNA breaks in breast cancer cells. BT-549 cells presented more TUNEL-positive cells than those of MCF-7, MDA-231, and the percentage of TUNELpositive BT-549 cells was approximately $35 \%$, and the percentage of MDA-231 cells was $5 \%(P<0.05)$ (Fig. 4b). Furthermore, the expression of ATM, ATR, and $\mathrm{Y}-\mathrm{H} 2 \mathrm{AX}$ in the MDA-231 and BT-549 cells further 
confirmed that the BT-549 cells generally had lower levels of ATM, ATR, and $\mathrm{Y}-\mathrm{H} 2 \mathrm{AX}$, indicating a failure in the DNA damage response (Fig. 4b).

Interestingly, the TUNEL assay also showed that the BT-549 cells presented TUNEL positivity not only in the nuclei but also in $30 \%$ of the MNs (Fig. 4b), while there were no differences in 53BP1 or Y-H2AX staining in the MNs among the BT-549, MDA-231 and/or MCF-7 cells (Supplementary Fig. S4a). However, in further DNA damage analysis, RPA2 (replication protein A2) and PICH (PLK1-interacting checkpoint helicase), the factors involved in DNA replication, were stained in breast cancer cells. Some MNs in the BT549 cells, but not MDA-231 and/or MCF-7 cells, exhibited bright foci with RPA2 or PICH staining (Fig. 4d, e), indicating DNA single or double breaks owing to DNA replication. Some MNs could be separately labeled by BrdU, indicating unscheduled DNA replication in the MNs (Fig. 4f). More interestingly, immunofluorescence staining of pHH3 (phosphorylated histone 3), a marker of chromatin condensation, could be detected in some MNs, especially in the BT-549 cells, similar to mitotic cells, and pHH3-stained MNs were usually Lamin B1 negative (Fig. 4f), which indicated that this DNA damage might be caused by a process similar to apoptosis in the MNs [22]. The results indicated that at least a portion of cytoplasmic DNA possibly came from the collapse or degradation of MNs.

Taken together, the results suggested that free cytoplasmic DNA was involved in the DNA damage response failure and its consequential $\mathrm{MN}$ formation, part of which underwent collapse owing to replication and DNA damage.

\section{Inhibition of DNA autophagy induced growth arrest or cell death of cancer cells}

The above results demonstrated that breast cancer cells presented high DNA autophagic activity, which raised the question of whether autophagy influences the biological activity of this kind of cancer cell.

To further determine the role of autophagy in breast cancer cells, we grew MCF-7, MDA-MB-231 and BT549 cells in the presence of bafilomycin $A 1$ at various concentrations $(0 \mathrm{nM}, 1 \mathrm{nM}, 5 \mathrm{nM}, 10 \mathrm{nM})$ for 72 hours, and the results showed that the growth of both the MDA-MB-231 and BT-549 cells was inhibited in a dose-dependent manner (Fig. 5a). However, the BT-549 cells showed cytotoxicity in the presence of $10 \mathrm{nM}$ bafilomycin A1. This finding was confirmed by time course analysis, in which the BT-549 cells with high autophagic activity died after treatment with $10 \mathrm{nM}$ bafilomycin $\mathrm{A} 1$ for 72 hours, while the MDA-MB-231 cells only showed growth inhibition (Fig. 5b).

Similarly, after further treatment of the MCF-7, MDA-MB-231 and BT-549 cells with different concentrations of $\mathrm{CQ}(0 \mu \mathrm{M}, 5 \mu \mathrm{M}, 50 \mu \mathrm{M}, 100 \mu \mathrm{M})$ for 72 hours, cell survival and growth were evaluated (Fig. $5 \mathrm{c})$.

The effects of inhibition of DNA autophagy on cell growth and survival of breast cancer cells were also clarified through silencing of either CGAS or SQSTM1 in the MCF-7, MDA-231 and BT-549 cells. The viability of the BT-549 cells was markedly reduced after either si-cGAS or si-SQSTM1 silencing. However, the MCF-7, MDA-231 and HeLa cells were not affected significantly (Fig. 5c). Similarly, the BT-549 cells, 
but not MDA-231 cells, were inhibited by treatment with the STING antagonist $\mathrm{H}-151(2 \mu \mathrm{M}, 10 \mu \mathrm{M}, 20 \mathrm{M})$ for 24 hours (Supplementary Fig. S5a).

To further clarify inhibition of autophagy to DNA autophagy under enhanced DNA damage, MCF-7, MDA231 , HeLa cells treated with Hydroxyurea $(0.5 \mathrm{mM})$ or Aphidicolin $(1 \mu \mathrm{M})$, which could not only induce cytoplasmic accumulation of CGAS/SQSTM1, but also became sensitive to the inhibitors of CQ $(50 \mu \mathrm{M})$ or BafilomycinA1 (10 nM) (Supplementary Fig. S5b, S5c, S5d).

To further expand the observation of DNA autophagic inhibition to cell activity, we screened a series of human cancer cells with immunofluorescence double-staining of cGAS and SQSTM1, and HCT116, 786-0, PC3M, and DU145 cells were screened. Three categories of cancer cells-cells with high cGAS and SQSTM1 (786-0 and PC3M), low CGAS and SQSTM1 (HeLa and HCT116), and high SQSTM1 but low cGAS (DU145)-were selected (Fig. 5e). We treated the 786-0 cells with strong cGAS and SQSTM1 expression levels with CQ $(50 \mu \mathrm{M})$ or bafilomycin A1 (10 nM) (Fig. $5 f)$. In contrast, the cells with low cGAS and SQSTM1 levels were treated with CQ $(50 \mu \mathrm{M})$ or bafilomycin A1 $(10 \mathrm{nM})$. Moreover, the cells with high SQSTM1 but low cGAS levels were treated with CQ $(50 \mu \mathrm{M})$ or bafilomycin A1 (10 nM) (Fig. $5 \mathrm{~g})$. The results proved that the growth or survival of cancer cells with high DNA autophagy was sensitive to autophagic inhibition.

To explore the cell death induced by DNA autophagic inhibition, we analyzed the CQ- or bafilomycin A1treated MCF-7, MDA-MB-231 and BT-549 cells, and the active form of caspase-3 was not detected by staining (Fig. 5h). Therefore, caspase-independent cell death (CICD) was investigated. Autophagy-related CICD could be achieved through lysosomal membrane permeabilization (LMP), but because CQ is an LMP inducer and bafilomycin A1 is an LMP inhibitor (data not shown), LMP-induced cell death could be ruled out. Alternatively, CICD is usually mediated by increasing ROS (reactive oxygen species) owing to mitochondrial outer-membrane permeabilization (MOMP). The production of ROS was measured in the presence of $\mathrm{CQ}$ and bafilomycin $\mathrm{A} 1$ by dihydroethidium (1 mM), and the results showed that either $\mathrm{CQ}$ or bafilomycin A1 could increase ethidium-stained BT-549 cells but only slightly affected the MDA-231 or MCF-7 cells (Fig. 5i), confirming that CQ or bafilomycin A1 treatment could induce caspase-independent cell death in the BT-549 cells.

The results demonstrated that DNA autophagy could be necessary for the survival of cancer cells by clearing cytoplasmic free DNA to protect against cell death.

\section{Discussion}

Autophagy is an important adaptive process to recycle substances or clear damaged organelles. For decades, autophagy has been thoroughly elucidated in terms of its process, forms, regulation and biological roles. DNA autophagy has also been identified, especially as an mechanism against exogenous invasion of organisms, and is considered an innate immune mechanism [23]. However, apparently, unlike that of other substances, autophagy of genomic DNA, the cellular genetic materials, is difficult to be considered for multicellular organisms. Nevertheless, in unicellular lower eukaryotes such as yeasts, DNA 
autophagy, in which cell nuclei can undergo autophagy by well-established regulatory pathways for nucleophagy through either piecemeal microautophagy of the nucleus (PMN) or late nucleophagy, has been identified [23]. Even entire nuclei could be degraded by macroautophagy in filamentous fungi. For mammalian cells, MN-related nucleophagy has been described occasionally, but mechanistically, its detailed process is still elusive. Mammalian cells have a nuclear lamina structure, which is different from that of lower eukaryotes, such as yeasts, with no comparable lamins or a fibrous nuclear envelope scaffold [23]. Nevertheless, nucleophagy is considered to play an important role in maintaining cellular genomic stability, detecting DNA damage, and regulating cellular apoptosis, as well as cellular senescence $[24,25]$. MN assays showed increased MN frequencies in breast cancer lymphocytes, which were correlated with the progression of breast cancer [26]. MNs are abnormal components that exists in the cytoplasm, independent of the nuclear nucleus. Autophagy also contributes to the elimination of MNs [23]. However, for most cancer cells, MNs can persist, indicating the general elimination of MNs by nucleophagy. In addition, similar to other studies, our investigations showed that cGAS and/or SQSTM1 could be detected in some MNs. However, the percentage of MNs with colocalization was low even in the highly abundant MNs in the BT-549 cells. Nucleophagy was not a prevalent event in cancer cells. A previous report showed the interaction between Lamin B1 and LC3 and suggesting that it is a nucleophagic mechanism (23). However, we still did not observe such interactions in our experiments, for instance, in some breast cell lines, such as BT-549, MDA-231, which usually showed a relatively high frequency of MN formation. Another possibility could not be ruled out: MNs undergoing nucleophagy could directly fuse with lysozyme, but this hypothesis needs to be further explored.

Selective DNA autophagy has been well clarified in mammalian cells. It has been demonstrated that free DNA or RNA could directly mediate microautophagy via LAMP2 (lysosome-associated membrane protein 2) without the need for LC3 or other autophagic factors, but the nucleic acid transporter SIDT2 (SID1 transmembrane family member 2 , SIDT2) is an integral lysosomal membrane protein for translocation into the lysosomal lumen. Nevertheless, specific DNA sensors, such as CGAS-STING, have also been revealed to participate in DNA autophagic initiation. CGAS binds to DNA (in MNs or free) and recruits Beclin-1 and STING, promoting autophagy. cGAS generates CGAMP and stimulates the STING-Golgi apparatus, but some studies have also shown that STING itself could mediate autophagy after its binding to DNA, and the intrinsic domains of STING could directly interact with LC3 [8]. In a recent report, exogenous plasmids were first recognized by DAI/ZBP1 (DNA-dependent activator of interferon regulatory factors/Z-DNA binding protein 1) but not cGAS [27]. However, in our study, upon introducing genomic DNA into the cancer cells, we found that cytosolic inclusions of various sizes were positive for CGAS, LC3 and lysosomes, confirming DNA autophagy existed in cancer cells (Supplementary Fig. S6). However, few inclusions were Beclin-1 positive (Supplementary Fig. S6). Therefore, it seemed that cytoplasmic DNA autophagy in cancer cells might involve different forms owing to the source of DNA and especially the DNA status and its level, nucleophagy, and selective autophagy. Apparently, the molecular mechanism could be diverse, such as LC3-dependent or LC3-independent DNA sensors. In actual conditions, free DNA is not naked but is instead usually bound to nuclear proteins. Therefore, genomic DNA from MN collapse or nuclear release might trigger a more complicated autophagic reaction to both DNA and proteins, especially ubiquitination (see below). 
In recent years, extensive research has demonstrated that SASP is activated by the cGAS-STING pathway, and its proinflammatory role has been demonstrated to be crucial for the occurrence of autoinflammatory disorders, age-related diseases and even cancer progression [4]. However, either cGAS or STING alone or their combination could mediate DNA autophagy [8]. The described data also indicated that cGAS could play an important role in autophagy. These findings raise an important question of how the decision to choose SASP or autophagy is made in cells. Our research showed that autophagy was usually found in breast cells with profound DNA damage such as the BT-549, while increased DNA damage in other cancer cells could induce DNA autophagic activity, indicating that the extent of DNA damage could be a factor influencing this determination. CGAS-mediated SASP and autophagy could respond to DNA damage. Severe DNA damage should be cleared by autophagy, but relatively less severe damage triggers SASP. Apparently, the cellular ability of DNA damage repair could also be a factor. These findings raise the question of whether there is any difference between cGAS in mediating SASP and autophagy. The details of cGAS recognition of DNA to induce autophagy or SASP are still unclear. However, more importantly, released genomic DNA from the cytoplasm is not protein-free but is instead bound with histones or other nuclear proteins [28]. The complex of DNA and protein could more easily activate autophagic activity since histones are usually ubiquitinated and generally recognizable by SQSTM1. To date, many studies have observed that SQSTM1 could be detected in the cell nucleus by either tagged SQSTM1 or immunohistochemistry [21]. In fact, the nuclear localization signal of SQSTM1 has been revealed, and its nuclear translocation has been demonstrated to be involved in the DNA damage response $[29,30]$. Therefore, the cytoplasmic or nuclear distribution of SQSTM1 could be similar. Thus, it is more likely that with severe DNA damage or repair failure, a relative amount of genomic DNA with coated proteins is released into the cytoplasm to trigger an autophagy-mediated clearance response.

Cytoplasmic DNA can easily be derived from mitochondria, organelles in the cytoplasm, but the mechanism by which genomic DNA accumulates in the cytoplasm is still unclear. MN formation is believed to be a major source since various reports have shown that MNs from some cancer cells are not intact in their nuclear lamina due to RB deficiency. Indeed, defects in nuclear membrane assembly, either aberrant nuclear pore complexes (NPCs) or nuclear lamina defects owing to lamina gene mutations, have been shown to result in nuclear irregularity, lobulation or MN formation to cause cellular senescence and the SASP [6]. More than $60 \%$ of MNs are disrupted, and then, damaged DNA is released [6, 31]. However, in our investigation, as well as a previous report, MNs formed in a variety of cancer cell lines, including breast, colorectal, cervical and kidney cancer cell lines, generally had intact nuclear lamina proteins, such as Lamin AVC or B or their receptor LBR, nuclear pore complex (Nup153), TPR (blanked protein), and integral membrane proteins (Sun2, nesprin2) [18]. Thus, nuclear rupture did not appear to be a prevalent event for cancer cells, and cytoplasmic DNA from MNs needs to be further explored. Similar to its main nucleus, MNs can undergo various activities, including replication, transcription and DNA damage. Chromothripsis has been demonstrated to be a consequence of DNA damage in MNs [10]. This research also showed that unscheduled DNA replication and DNA damage could be detected in a portion of the MNs. Interestingly, some BT-549 cells with abundant MNs more frequently presented condensed focal staining of RPA2 or PICH, both of which participate in the DNA damage response; the former usually binds to single-stranded DNA, and the latter binds to double-stranded DNA [33, 34]. More importantly, some MNs 
were frequently observed in pHH3-positive cells, similar to BT-549 cells (Fig. 4f). It has been acknowledged that $\mathrm{pHH} 3$ is mainly found during chromatin condensation in mitosis and in apoptotic nuclei of cells [35, 36]. For determination of whether pHH3-positive MNs undergo mitosis, cells were stained for Hec1, a protein involved in kinetochore assembly. The results proved that no MNs were positive, but only mitotic and apoptotic cells were stained (data not shown). In a cell-free apoptotic model, nuclear condensation could sequentially proceed with condensation, a nuclear necklace, collapse or disassembly [22, 37]. Similarly, pHH3-stained MNs also presented these morphological changes (Fig. 4f), indicating that MNs could undergo collapse owing to replication and damage in breast cancer cells with DNA autopagy. Accordingly, a portion of the MNs in the BT-549 cells showed positive staining of RPA2 and PICH, especially in the foci-staining pattern (Fig. 4d, e), indicating severe DNA damage in these MNs. Apparently, the more MNs formed, the more frequently breakage was detected. In addition, the release of nuclear eccrDNA (extrachromosomal circular rDNA) should be another source of cytoplasm (see below).

Since $\mathrm{MN}$ formation can be generally induced by a variety of genotoxic agents, DNA damage is reasonably considered a key process, and aberrant mitosis is widely accepted [6, 12]. However, recent studies have suggested that for cancer cells, DNA replication stress could be a likely common mechanism $[33,38,39]$. Oncogenic mutations induce accelerated DNA replication and trigger replication stress. The so-called common fragile sites in the genome, such as rDNA, are difficult to replicate, and stalled or collapsed replication forks usually induce the formation of UFBs (ultrafine bridges) or lagging chromosomes to result in MN formation or to generate free DNA fragments, such as eccrDNA, which are hard to enclose in the late phase of mitosis during nuclear membrane assembly and are consequently released to the cytoplasm [16, 34]. In our investigation, in addition to MNs, cytoplasmic DNA from the genome, including Alu-repeated sequences and rDNA, was easily detected, indicating a replication stressrelated mechanism. Moreover, intra-S phase checkpoints mediated by ATR and ATM kinases are crucial to replication stress, and their deficiency causes replication stress-related DNA damage [40]. The BT-549 cells generally had low levels of pATM and pATR and high formation of MNs and cytoplasmic DNA as well as strong TUNEL staining, suggesting relationships between these factors.

Autophagy is a form of cellular activity that adapts to endogenous and environmental changes. Although gene mutations related to the regulation of autophagy have been clarified in tumorigenesis, for some kinds of cancer cells, inhibition of autophagy could promote their growth and survival, indicating that autophagic activity could be necessary for these cancer cells [41]. However, how to determine the sensitivity of cancers to autophagic inhibition is still not established. This investigation mainly showed that inhibition of DNA autophagy could decrease cell viability in breast cancer cells even more cancer cells, suggesting its potential therapeutic utility in cancer treatment, especially for cancer cells deficient in DNA repair. In recent years, DNA damage repair deficiency has been successfully used in cancer therapy; for instance, cancers with MSI (microsatellite instability) can be treated with immune checkpoint blockadebased immunotherapies, while genomic mutations of BRCA1/2 or HRR (homologous recombination repair) are targets of PARP inhibitors. DDR deficiency has been considered a promising anticancer target [42-44]. Targeting autophagy could be another approach to treat DDR-deficient cancers. 


\section{Conclusion}

In summary (Fig. 6), our investigation revealed DNA autophagy in breast cancer cells with high MN formation. Autophagy of genomic DNA in the cytosol could be mediated by cGAS but is usually coordinated with other autophagic mediators. Cytoplasmic DNA could be derived from DNA replicationinduced damage and MN collapse. The clearance of cytoplasmic DNA could be necessary for cancer cell growth and survival. Thus, autophagic inhibition could be a potential therapeutic approach for cancer cells especially in breast cancer cells with high DNA autophagic activity.

\section{Abbreviations}

MN: micronuclei; SASP: senescence-associated secretory phenotype; cGAS: GMP-AMP synthase; STING: stimulate stimulator of interferon gene; BA1: Bafilomycin A1; CICD: caspase-independent cell death; LMP: lysosomal membrane permeabilization; SA- $\beta$-gal: Senescence associated- $\beta$-galactosidase staining; FISH: Fluorescence in situ hybridization; RPA2: replication protein A2

pHH3: phosphorylated histone 3; PICH: PLK1-interacting checkpoint helicase; LAMP2: lysosomeassociated membrane protein 2; SIDT2:SID1 transmembrane family member 2, SIDT2; eccrDNA : extrachromosomal circular rDNA囚MSIØmicrosatellite instability囚HRR囚homologous recombination repair[

\section{Declarations}

Ethics approval and consent to participate

Not applicable.

Consent for publication

Not applicable.

Availability of data and materials

All data generated or analyzed during this study are included in this published article and its supplementary information files.

Competing interests

The authors have declared that no competing interests exist.

Funding

This project was supported by the National Natural Science Foundation of China (No. 81872018) and the Key Project from the Chinese Ministry of Science and Technology (No. 2017YFC0110200). 
Mengfei Yao, Yaqian Wu performed experiments and analyzed the data; Yanan Cao, Ningning Ma, Yijie Chai conducted the statistical analyses; Bo Zhang and Mengfei Yao designed the study and wrote the manuscript; Yaqian Wu and Haijing Liu revised manuscript. Hong Zhang, Shuang Zhang, Lin Nong and Li Liang assisted some experiments. All authors read and approved the final manuscript.

Acknowledgement

We thank Dr. Dawei Xu and Cheng Liu for their kind suggestions to the manuscript and appreciate Mr. Hongquan Shao and Mr. Ning Li for helpful technique support.

Authors' information

${ }^{1}$ Department of Pathology, Peking University Health Science Center, Beijing 100191, China.

${ }^{2}$ Department of Pathology, Peking University First Hospital, Beijing 100034, China.

\section{References}

1. Ablasser A, Goldeck M, Cavlar T, Deimling T, Witte G, Röhl I, et al. cGAS produces a 2'-5'-linked cyclic dinucleotide second messenger that activates STING. Nature. 2013;498(7454):380-4.

2. Chen Q, Sun L, Chen ZJ. Regulation and function of the cGAS-STING pathway of cytosolic DNA sensing. Nature immunology. 2016;17(10):1142-9.

3. Coppé JP, Patil CK, Rodier F, Sun Y, Muñoz DP, Goldstein J, et al. Senescence-associated secretory phenotypes reveal cell-nonautonomous functions of oncogenic RAS and the p53 tumor suppressor. PLoS Biol. 2008;6(12):2853-68.

4. Sun L, Wu J, Du F, Chen X, Chen ZJ. Cyclic GMP-AMP synthase is a cytosolic DNA sensor that activates the type I interferon pathway. Science. 2013;339(6121):786-91.

5. Loo TM, Miyata K, Tanaka Y, Takahashi A. Cellular senescence and senescence-associated secretory phenotype via the cGAS-STING signaling pathway in cancer. Cancer Sci. 2020;111(2):304-11.

6. Li T, Chen ZJ. The cGAS-cGAMP-STING pathway connects DNA damage to inflammation, senescence, and cancer. The Journal of experimental medicine. 2018;215(5):1287-99.

7. Hinds P, Pietruska J. Senescence and tumor suppression. F1000Research. 2017;6:2121.

8. Gui X, Yang H, Li T, Tan X, Shi P, Li M, et al. Autophagy induction via STING trafficking is a primordial function of the cGAS pathway. Nature. 2019;567(7747):262-6.

9. Li X, Shu C, Yi G, Chaton CT, Shelton CL, Diao J, et al. Cyclic GMP-AMP synthase is activated by double-stranded DNA-induced oligomerization. Immunity. 2013;39(6):1019-31.

10. Kisurina-Evgenieva OP, Sutiagina OI, Onishchenko GE. Biogenesis of Micronuclei. Biochemistry Biokhimiia. 2016;81(5):453-64.

11. Jdey W, Thierry S, Popova T, Stern MH, Dutreix M. Micronuclei Frequency in Tumors Is a Predictive Biomarker for Genetic Instability and Sensitivity to the DNA Repair Inhibitor AsiDNA. Cancer research. 
2017;77(16):4207-16.

12. Lewis CW, Golsteyn RM. Cancer cells that survive checkpoint adaptation contain micronuclei that harbor damaged DNA. Cell cycle (Georgetown Tex). 2016;15(22):3131-45.

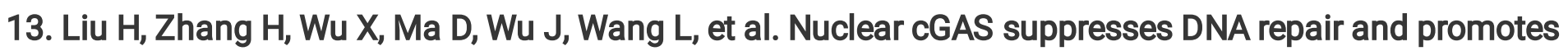
tumorigenesis. Nature. 2018;563(7729):131-6.

14. Mackenzie KJ, Carroll P, Martin CA, Murina O, Fluteau A, Simpson DJ, et al. cGAS surveillance of micronuclei links genome instability to innate immunity. Nature. 2017;548(7668):461-5.

15. Bakhoum SF, Ngo B, Laughney AM, Cavallo JA, Murphy CJ, Ly P, et al. Chromosomal instability drives metastasis through a cytosolic DNA response. Nature. 2018;553(7689):467-72.

16. Liao Z, Jiang W, Ye L, Li T, Yu X, Liu L. Classification of extrachromosomal circular DNA with a focus on the role of extrachromosomal DNA (ecDNA) in tumor heterogeneity and progression. Biochimica et biophysica acta Reviews on cancer. 2020;1874(1):188392.

17. Banoth B, Cassel SL. Mitochondria in innate immune signaling. Translational research: the journal of laboratory clinical medicine. 2018;202:52-68.

18. Cao Y, Yao M, Wu Y, Ma N, Liu H, Zhang B. N-Acetyltransferase 10 Promotes Micronuclei Formation to Activate the Senescence-Associated Secretory Phenotype Machinery in Colorectal Cancer Cells. Transl Oncol. 2020;13(8):100783.

19. Fernandez-Martinez J, LaCava J, Rout MP. Density Gradient Ultracentrifugation to Isolate Endogenous Protein Complexes after Affinity Capture. Cold Spring Harb Protoc 2016;2016(7).

20. Murray K. The acid extraction of histones from calf thymus deoxyribonucleoprotein. Journal of molecular biology. 1966;15(2):409-19.

21. Wang Y, Zhang N, Zhang L, Li R, Fu W, Ma K, et al. Autophagy Regulates Chromatin Ubiquitination in DNA Damage Response through Elimination of SQSTM1/p62. Molecular cell. 2016;63(1):34-48.

22. Toné S, Sugimoto K, Tanda K, Suda T, Uehira K, Kanouchi H, et al. Three distinct stages of apoptotic nuclear condensation revealed by time-lapse imaging, biochemical and electron microscopy analysis of cell-free apoptosis. Experimental cell research. 2007;313(16):3635-44.

23. Mijaljica D, Devenish RJ. Nucleophagy at a glance. Journal of cell science. 2013;126(Pt 19):4325-30.

24. Bo Otto F, Thumm M. Nucleophagy-Implications for Microautophagy and Health. International journal of molecular sciences 2020;21(12).

25. Fu N, Yang X, Chen L. Nucleophagy Plays a Major Role in Human Diseases. Curr Drug Targets. 2018;19(15):1767-73.

26. Baeyens A. Chromosomal radiosensitivity of lymphocytes in South African breast cancer patients of different ethnicity: An indirect measure of cancer susceptibility. South African medical journal = SuidAfrikaanse tydskrif vir geneeskunde. 2015;105(8):675-8.

27. Semenova N, Bosnjak M, Markelc B, Znidar K, Cemazar M, Heller L. Multiple cytosolic DNA sensors bind plasmid DNA after transfection. Nucleic acids research. 2019;47(19):10235-46. 
28. Giné E, Crespo M, Muntañola A, Calpe E, Baptista MJ, Villamor N, et al. Induction of histone H1.2 cytosolic release in chronic lymphocytic leukemia cells after genotoxic and non-genotoxic treatment. Haematologica. 2008;93(1):75-82.

29. Kang C, Elledge SJ. How autophagy both activates and inhibits cellular senescence. Autophagy. 2016;12(5):898-9.

30. Feng Y, Klionsky DJ: Autophagy regulates DNA repair through SQSTM1/p62. Autophagy 2017;13(6):995-996.

31. Hatch EM, Fischer AH, Deerinck TJ, Hetzer MW. Catastrophic nuclear envelope collapse in cancer cell micronuclei. Cell. 2013;154(1):47-60.

32. Maass KK, Rosing F, Ronchi P, Willmund KV, Devens F, Hergt M, et al. Altered nuclear envelope structure and proteasome function of micronuclei. Experimental cell research. 2018;371(2):353-63.

33. Gelot C, Magdalou I, Lopez BS. Replication stress in Mammalian cells and its consequences for mitosis. Genes. 2015;6(2):267-98.

34. Bjerregaard VA, Özer Ö, Hickson ID, Liu Y: The Detection and Analysis of Chromosome Fragile Sites. Methods in molecular biology (Clifton, NJ) 2018;1672:471-482.

35. Webster PJ, Littlejohns AT, Gaunt HJ, Prasad KR, Beech DJ, Burke DA. AZD1775 induces toxicity through double-stranded DNA breaks independently of chemotherapeutic agents in p53-mutated colorectal cancer cells. Cell cycle (Georgetown Tex). 2017;16(22):2176-82.

36. Pérez-Cadahía B, Drobic B, Davie JR. H3 phosphorylation: dual role in mitosis and interphase. Biochemistry and cell biology = Biochimie. et biologie cellulaire. 2009;87(5):695-709.

37. Crowley LC, Marfell BJ, Waterhouse NJ. Analyzing Cell Death by Nuclear Staining with Hoechst 33342. Cold Spring Harb Protoc 2016; 2016(9).

38. Wilhelm T, Olziersky AM, Harry D, De Sousa F, Vassal H, Eskat A, et al. Mild replication stress causes chromosome mis-segregation via premature centriole disengagement. Nature communications. 2019;10(1):3585.

39. Sabatinos SA, Ranatunga NS, Yuan JP, Green MD, Forsburg SL. Replication stress in early S phase generates apparent micronuclei and chromosome rearrangement in fission yeast. Molecular biology of the cell. 2015;26(19):3439-50.

40. Maréchal A, Zou L. DNA damage sensing by the ATM and ATR kinases. Cold Spring Harbor perspectives in biology 2013;5(9).

41. Kimmelman AC, White E. Autophagy and Tumor Metabolism. Cell Metabol. 2017;25(5):1037-43.

42. Hengel SR, Spies MA, Spies M. Small-Molecule Inhibitors Targeting DNA Repair and DNA Repair Deficiency in Research and Cancer Therapy. Cell chemical biology. 2017;24(9):1101-19.

43. Goyal G, Fan T, Silberstein PT. Hereditary cancer syndromes: utilizing DNA repair deficiency as therapeutic target. Familial cancer. 2016;15(3):359-66.

44. Dudley JC, Lin MT, Le DT, Eshleman JR. Microsatellite Instability as a Biomarker for PD-1 Blockade. Clinical cancer research: an official journal of the American Association for Cancer Research. 2016;22(4):813-20. 


\section{Figures}

Figure 1

A
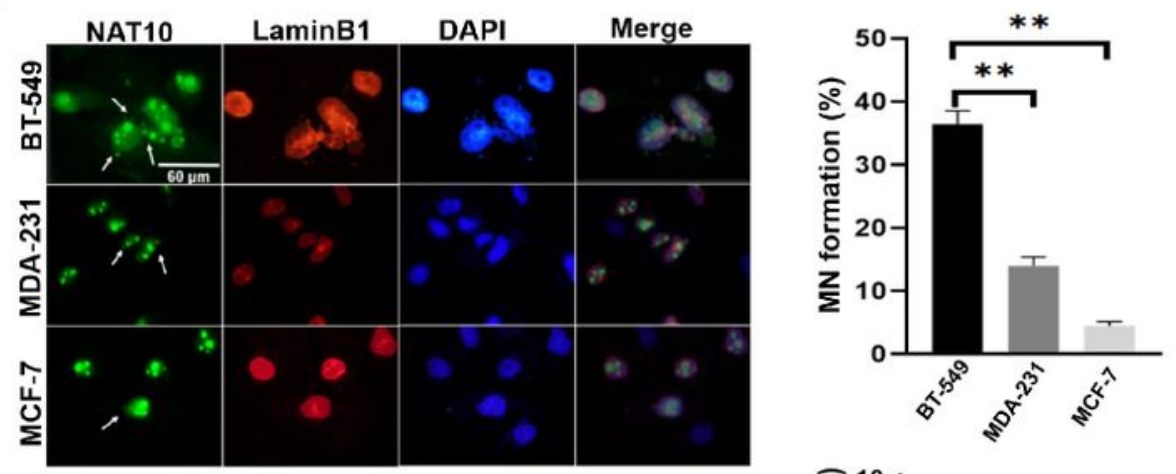

B
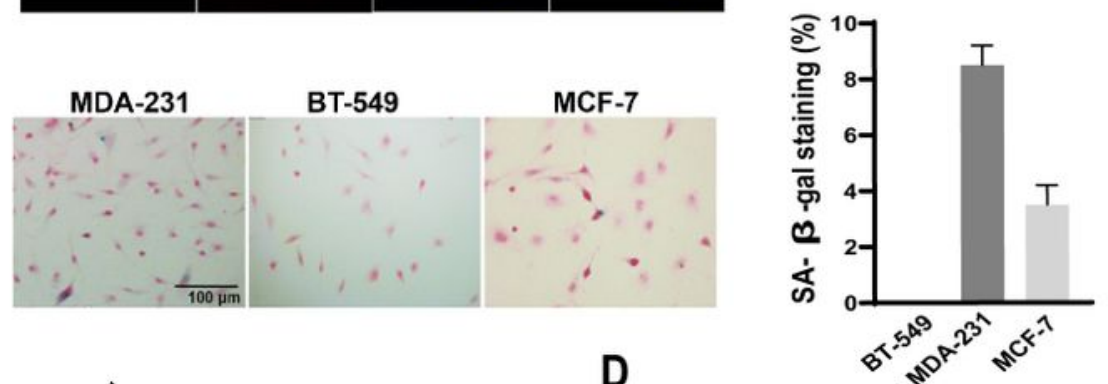

C

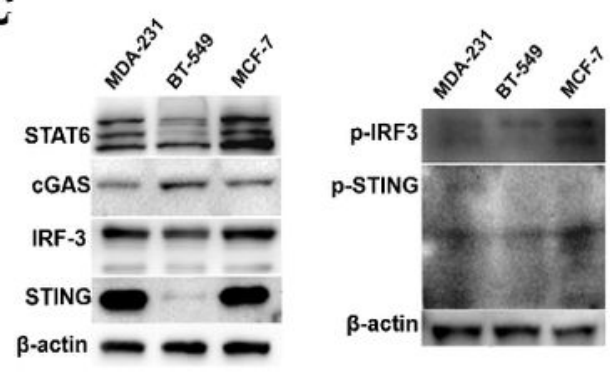

D

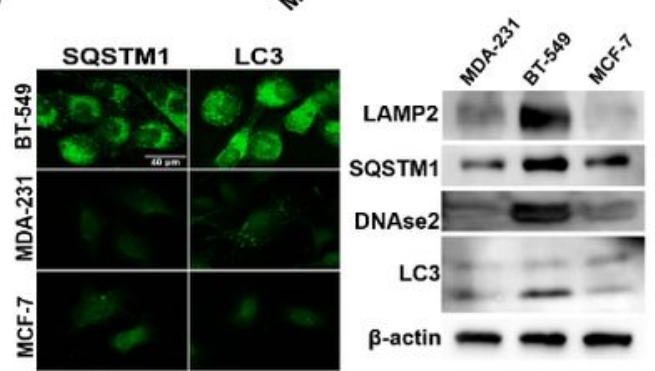

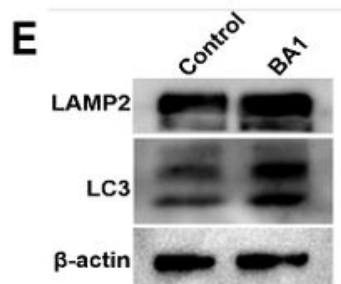

$\mathbf{F}$

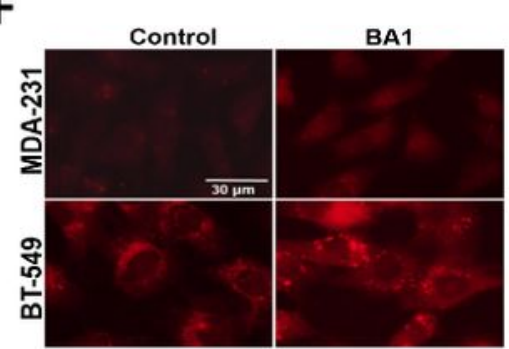

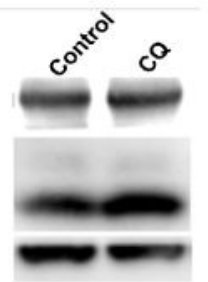

G

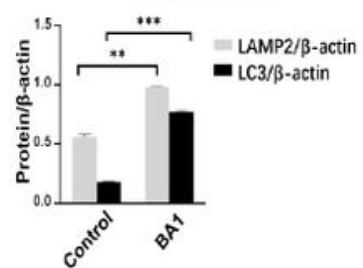

G

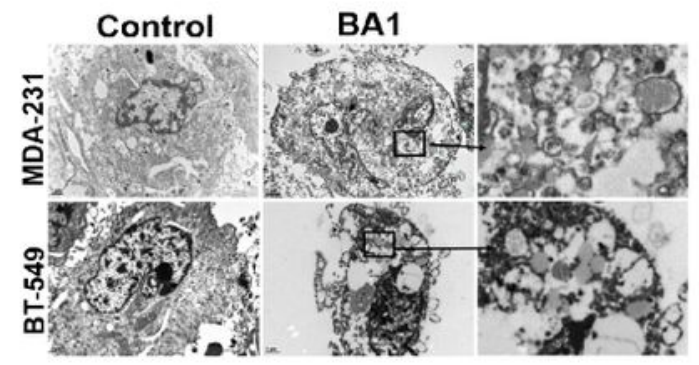

Figure 1

High frequency of MNs in breast cancer cells was associated with an autophagic phenotype. a MN formation in breast cancer cells. Left panels: MDA-231, BT-549 and MCF-7 cells were subjected to immunofluorescence staining for NAT10 and Lamin B1. Arrows indicate MNs. Right panel: The frequency 
of MNs in each kind of cell was counted and calculated as the percentage of total cells. b Senescent phenotypes of breast cancer cells. Left panels: Representative images of SA- $\beta$-gal staining in the MDA231, BT549 and MCF7 cells. Right panel: SA- $\beta$-gal-positive cells were counted and calculated as the percentage of total cells. $c$ The expression of SASP-related factors in breast cancer cells. The MDA-231, BT549 and MCF-7 cells were assessed by Western blotting. Left panels: the levels of STAT6, cGAS, STING, and IRF3. Right panels: p-STING and p-IRF3. $d$ The expression of autophagy-related proteins in the MDA231, BT-549 and MCF-7 cells. Left panels: The cells were subjected to immunofluorescence staining of SQSTM1 or LC3. Right panels: Total cell proteins were extracted, and LAMP2, SQSTM1, DNase II and LC3 were measured by Western blotting. $\mathrm{e}$ The analysis of autophagic flow in the BT-549 cells. The BT-549 cells were treated with $50 \mu \mathrm{M}$ CQ for 4 hours or $10 \mathrm{nM}$ bafilomycin A1 for 24 hours, and total cell proteins were extracted and analyzed by Western blotting. Left panel: The levels of LAMP2 and LC3 in the CQtreated cells. Right panel: The levels of SQSTM1 and LC3 in the bafilomycin A1-treated cells. The plots represent quantitative calculations of the corresponding left or right panels. $f$ The activity of lysosomes in the MDA-231 and BT-549 cells. After treatment with $10 \mathrm{nM}$ bafilomycin A1 (BA1) for 24 hours, the cells were stained with LysoTracker. $g$ Electron microscopy of the autophagic activity in the MDA-231 and BT549 cells. After treatment with bafilomycin A1 (BA1) for 72 hours, the cells were subjected to transmission electron microscopy. $\beta$-actin was used as an internal standard in Western blotting. The level of statistical significance was $<0.05\left(^{(}\right),<0.01\left(^{(*)}\right)$, and $<0.001\left(^{(* *)}\right.$. The experiments were independently repeated, and the images are representative of repeated experiments. 
Figure 2

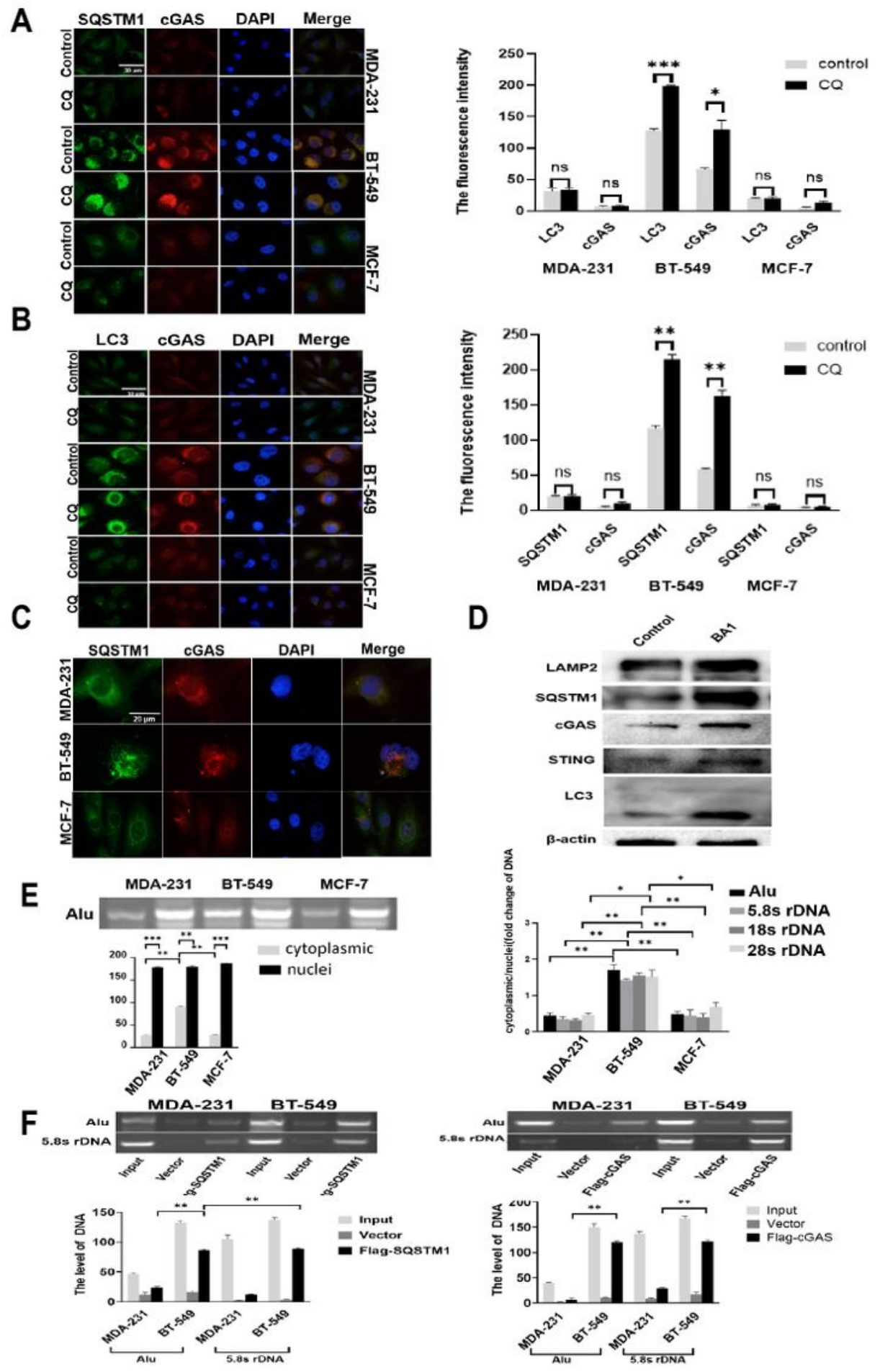

Figure 2

Detection of genomic DNA autophagy in the cytoplasm of breast cancer cells. a The effects of autophagic inhibition on the expression and distribution of SQSTM1 and CGAS in breast cancer cells. MDA-231 and BT-549 cells were treated with CQ $(10 \mu \mathrm{M})$ for 4 hours and subjected to double immunofluorescence staining of SQSTM1 (green) and cGAS (red). b The effects of autophagic inhibition on the expression and distribution of LC3 and cGAS in breast cancer cells. The MDA-231 and BT-549 cells were treated with CQ 
$(10 \mu \mathrm{M})$ for 4 hours and subjected to double immunofluorescence staining for LC3 (green) and cGAS (red). c Detection of SQSTM1 and cGAS in the MNs of breast cancer cells. The MDA-231, BT-549 and MCF-7 cells were doubly stained for SQSTM1 (green) and CGAS (red) by immunofluorescence, and MN colocalization is labeled with circles. $d$ Autophagic inhibition increased the level of cGAS in the BT-549 cells. The BT-549 cells were treated with bafilomycin A1 (BA1, $10 \mathrm{nM}$ ) for 24 hours, and the levels of cGAS, SQSTM1, STING, LAMP2, and LC3 were measured by Western blotting. e Analysis of cytoplasmic DNA in breast cancer cells. The cytoplasmic or nuclear DNA of the MDA-231, BT-549 and MCF-7 cells was independently isolated, and Alu- and rDNA (5.8S, 18S and 28S) were individually detected by PCR from cytoplasmic or nuclear DNA, respectively, as described in the Materials and Methods. Left panels: The amplified Alu products are displayed by gel electrophoresis. Right panel: The summarized results of rDNA (5.8S, $18 S$ and $28 S$ ) in real-time PCR. $f$ Immunoprecipitation analysis of genomic DNA and cGAS or SQSTM1 in the cytoplasm. The BT-549 cells and the MDA-231 cells were transfected with Flag-cGAS or Flag-SQSTM1 (or vector) for 36 hours. After fixation with $1 \%$ formalin for 10 minutes, cytoplasmic extracts were isolated as described in the Materials and Methods. The genomic DNA was measured by PCR amplification of Alu- and 5.8S rDNA. Left panels: Flag-SQSTM1. Right panels: Flag-cGAS. The quantitative plots were calculated from the corresponding gel electrophoresis results. $\beta$-actin was used as an internal standard in Western blotting. The level of statistical significance was $<0.05(*),<0.01(* *),<0.001(* *)$, ns: no significance. All experiments were repeated three times independently, and the images are representative of repeated experiments. 
Figure 3
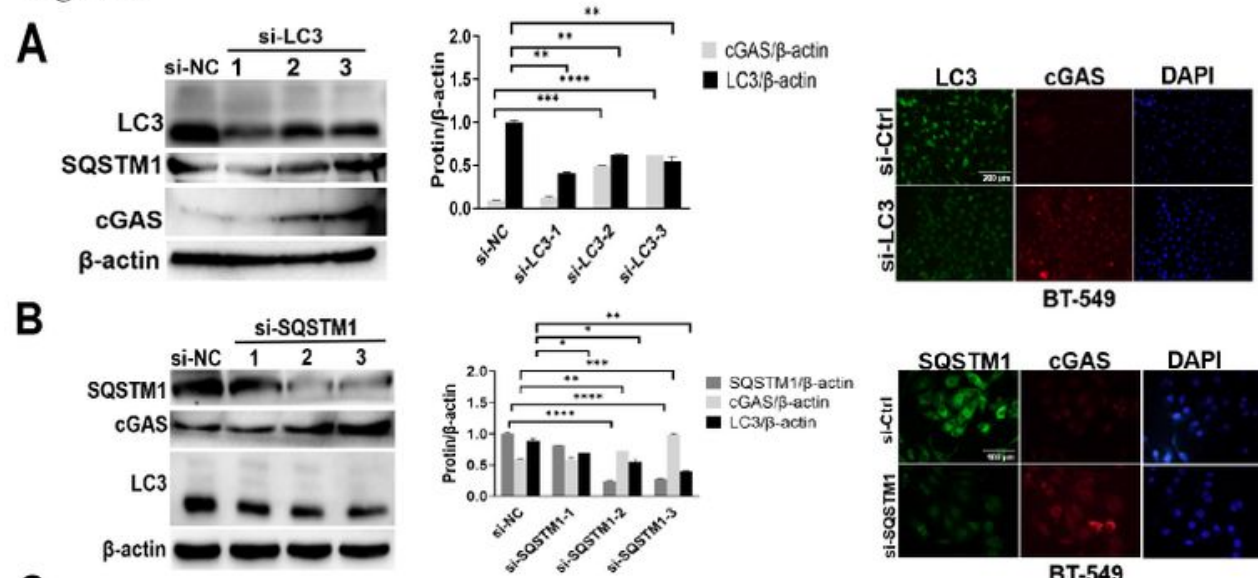

C
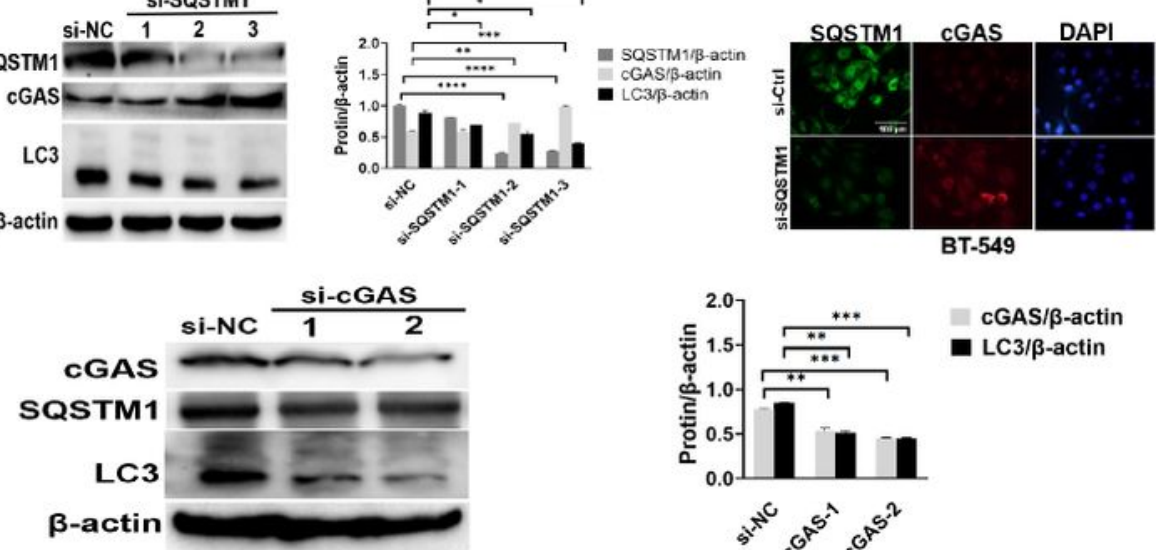

D
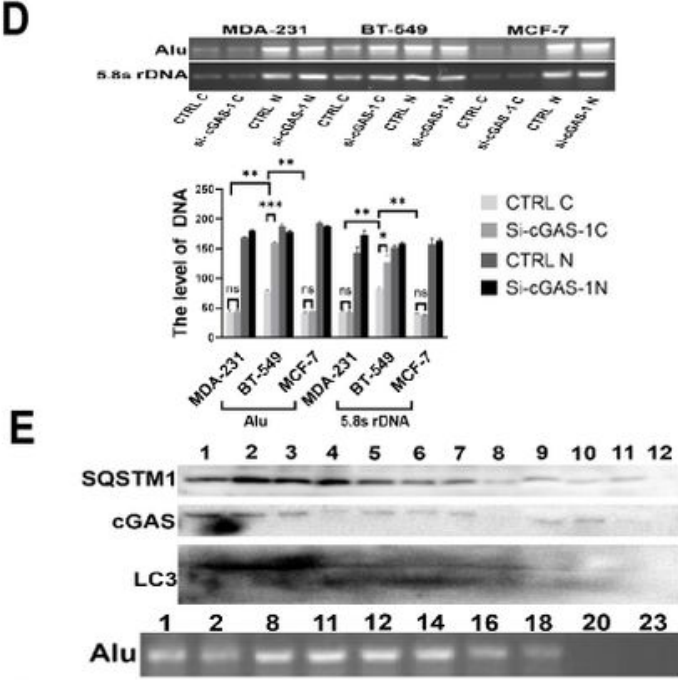

E
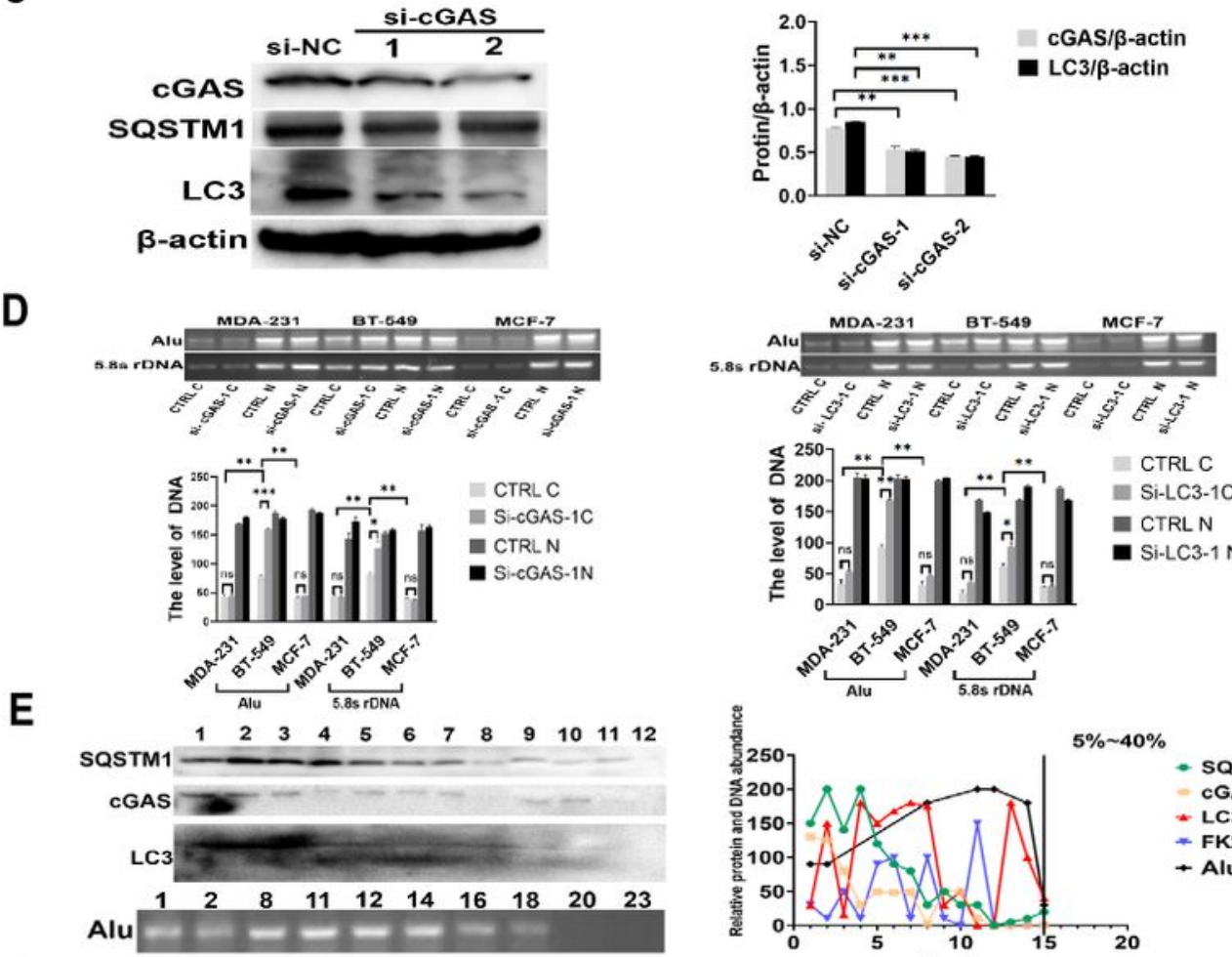

드 CTRL C
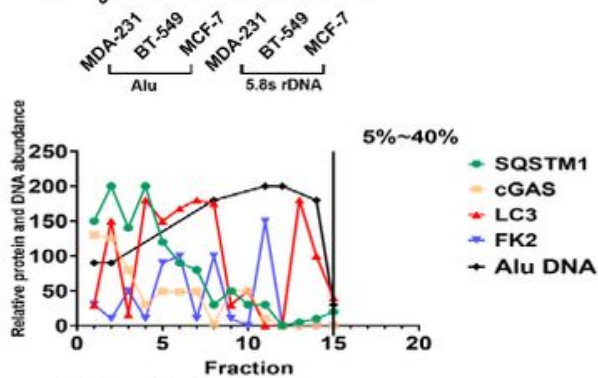

F
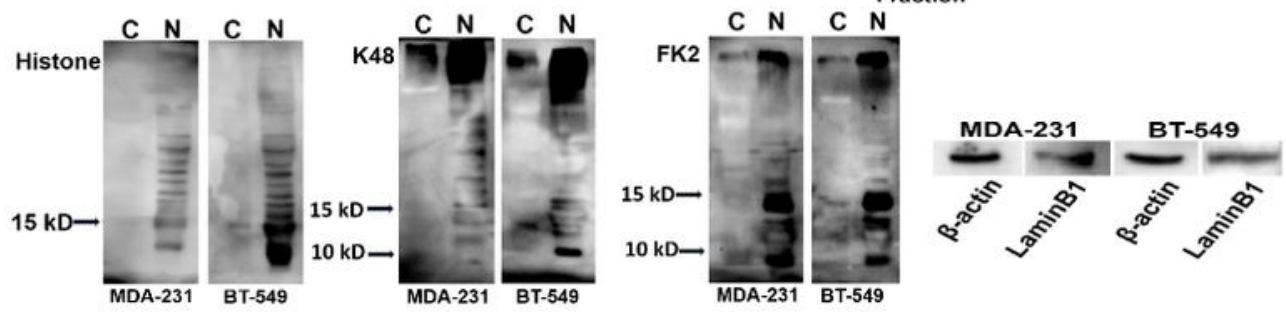

Figure 3

DNA autophagy in the cytoplasm was involved in the coordination of CGAS and SQSTM1. a The depletion

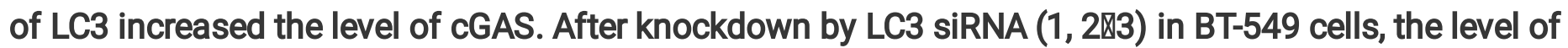
cGAS was measured by Western blotting (left panels), while the cellular distribution of LC3 (green) and cGAS (red) in the LC3 siRNA (3)-transfected cells was analyzed by immunofluorescence staining (right panels). siNC: control iRNA. b The depletion of SQSTM1 increased the level of cGAS but decreased LC3. 
After knockdown of SQSTM1 by siRNA $(1,2,3)$ in BT-549 cells, the levels of cGAS or LC3 were measured by Western blotting (left panels), and the cellular distribution of cGAS and LC3 in the SQSTM1 siRNA (3)transfected cells was analyzed by immunofluorescence staining (right panels). siNC: control iRNA. c The depletion of cGAS decreased the level of LC3. After knockdown by cGAS siRNA $(1,2)$ in BT-549 cells, the levels of LC3 or SQSTM1 were measured by Western blotting. siNC: control iRNA. $d$ The silencing of autophagic genes increased the level of cytoplasmic DNA. Cytoplasmic or nuclear DNA was extracted from the MCF-7, MDA-231 and BT-549 cells or their corresponding cells with knockdown of SQSTM1 or LC3 by siRNA. The levels of cytoplasmic (C) or nuclear (N) DNA were measured by PCR amplification of Alu and 5.8S rDNA sequences, and the quantitative analysis was performed with the results of gel electrophoresis. e Sucrose density gradient analysis of the cytoplasmic fractions of the BT-549 cells. Cytoplasmic extracts from the BT-549 cells were isolated and fractioned through sucrose gradient centrifugation, and SQSTM1, CGAS, and LC3 in each fraction were measured by Western blotting. Cytoplasmic genomic DNA in selected fractions was analyzed by Alu-based PCR amplification and gel electrophoresis. The amounts of the indicated proteins were quantified using ImageJ and plotted. $f$ Analysis of cytoplasmic histones and ubiquitination in breast cancer cells. Cytoplasmic or nuclear extracts from the MDA-231 or BT-549 cells were independently isolated by acid extraction and analyzed by Western blotting. Left panels: Core histone; middle panels: K48 antibody detection; right panels: FK2 antibody detection of mono- or polyubiquitinated proteins. The arrows indicate the putative histones. C: cytoplasmic; N: nuclear. The level of statistical significance was $<0.05\left({ }^{*}\right),<0.01(* *)$, and $<0.001$ (**). All the experiments were independently repeated, and the images are representative of repeated experiments. 
Figure 4

A

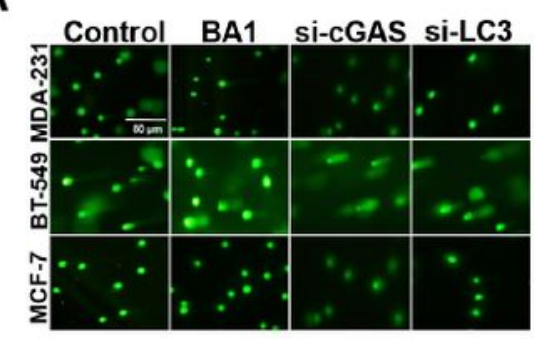

B
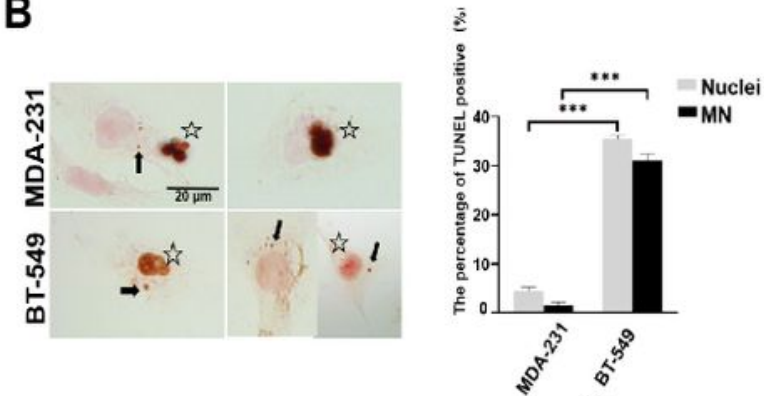

C

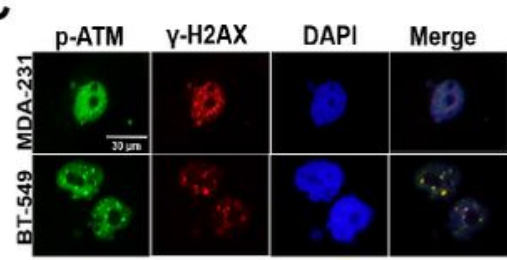

D

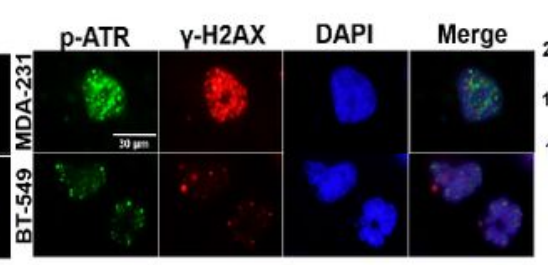

E

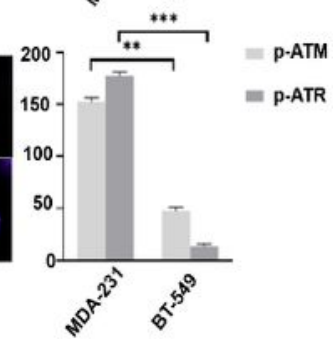

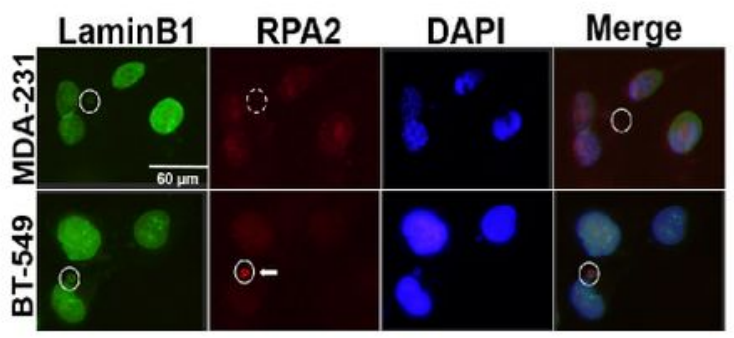

G

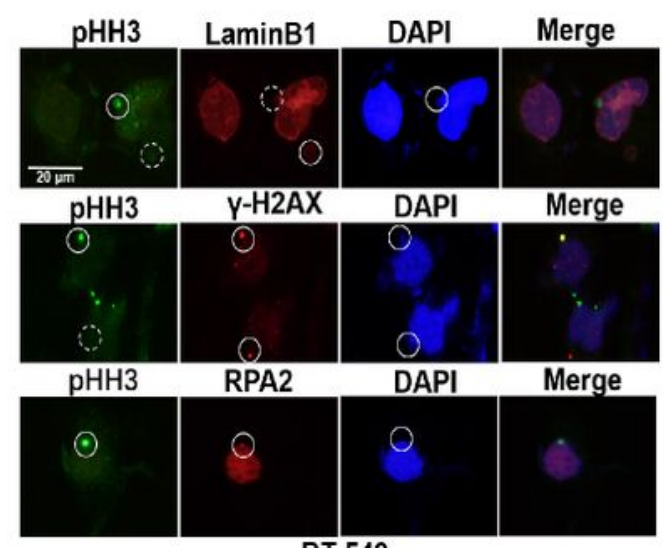

BT-549

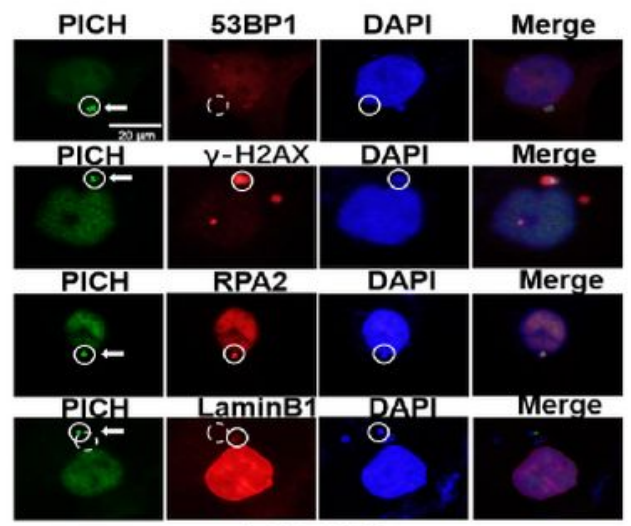

BT-549

F

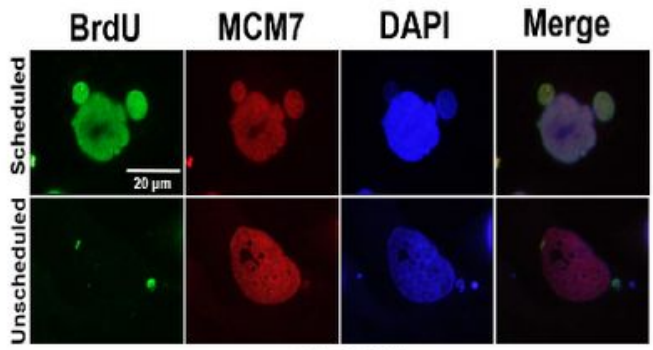

BT-549

Figure 4

Genomic DNA in the cytoplasm could be derived from either damaged nuclei or MNs. a Comet assays of the status of DNA damage in breast cancer cells. The original MDA-231, BT-549 and MCF-7 cells, those in the presence of $10 \mathrm{nM}$ BAI (bafilomycin A1) for 24 hours, or cells with silencing of cGAS or LC3 by transfection with either si-cGAS or si-LC3 for 48 hours were subjected to comet assays. Their more detailed data are summarized in Table 1. b TUNEL analysis of breast cancer cells. A TUNEL assay was 
carried out on the MDA-231 and BT-549 cells, and positive staining in the nucleus and/or MNs was evaluated in whole slides and is presented as percentages. Arrows indicate positive MNs, while stars label positive nuclei. $c$ The status of DNA damage in breast cancer cells. The MDA-231 and BT-549 cells were stained for $\mathrm{Y}-\mathrm{H} 2 \mathrm{AX}$ and $\mathrm{p}-\mathrm{ATM}$ or $\mathrm{p}$-ATR, respectively (left panels). The staining intensity was evaluated and plotted (right panel). d Double staining of RPA2 and Lamin B1 was performed in the MDA-231 and BT549 cells. Solid circles label positive staining, and dashed circles indicate weak or negative staining. Arrow indicates RPA2 foci in MNs. e Double staining of PICH and RPA2 or 53BP-1 or Y-H2AX or Lamin B1 was performed in the BT-549 cells. Solid circles indicate positive staining, and dashed circles indicate weak or negative staining. Arrows indicate PICH foci in MNs. $f$ BrdU incorporation assays. After incubation with BrdU for 30 minutes, the BT-549 cells were doubly stained with BrdU and MCM7. Arrows indicate unscheduled replication in MNs. $g$ Analysis of DNA damage in the MNs in breast cancers. Double staining of Lamin B1, RPA2 or Y-H2AX and PHH3 was performed in the BT-549 cells. Solid circles indicate highly stained MNs, and dashed circles indicate negative or weak staining of MNs. The level of statistical significance was $<0.01(* *),<0.001(* \star *)$. The images are representative of repeated experiments. 
Figure 5

A

B
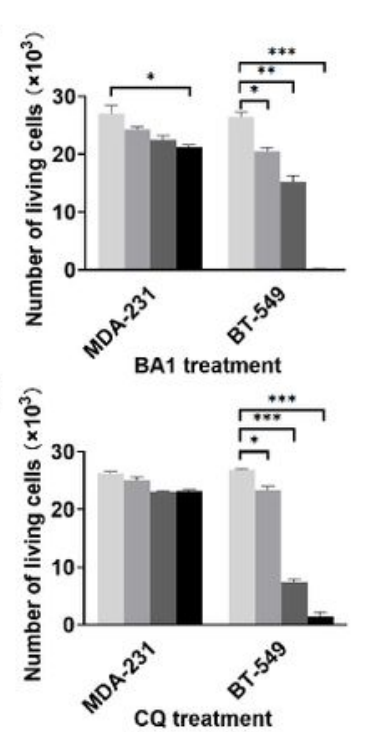

C

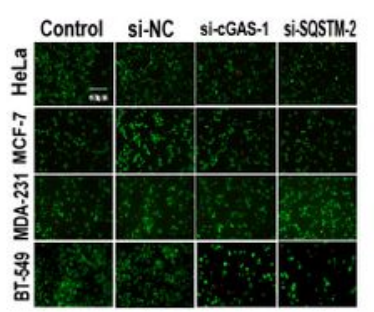

E

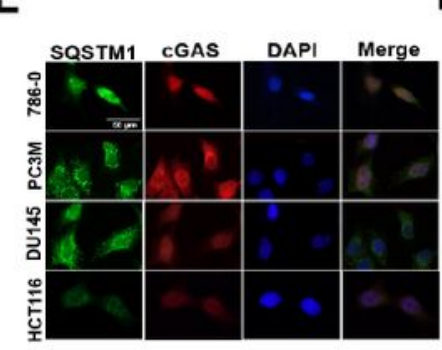

H

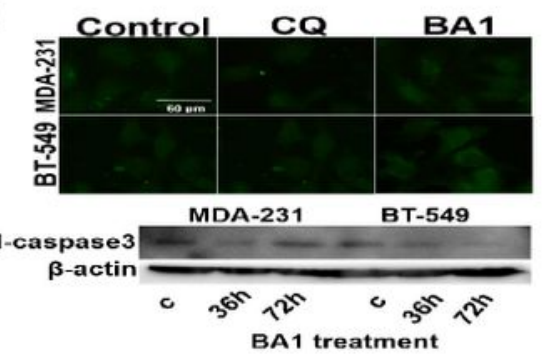

Control

- $5 \mu \mathrm{M}$

$50 \mu \mathrm{M}$

- 100 $\mathrm{\mu m}$

F
ติ
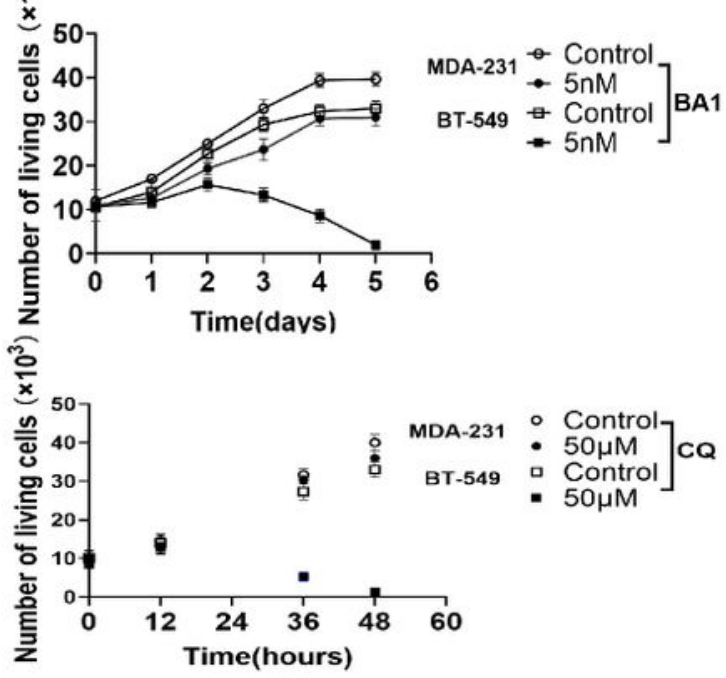

D
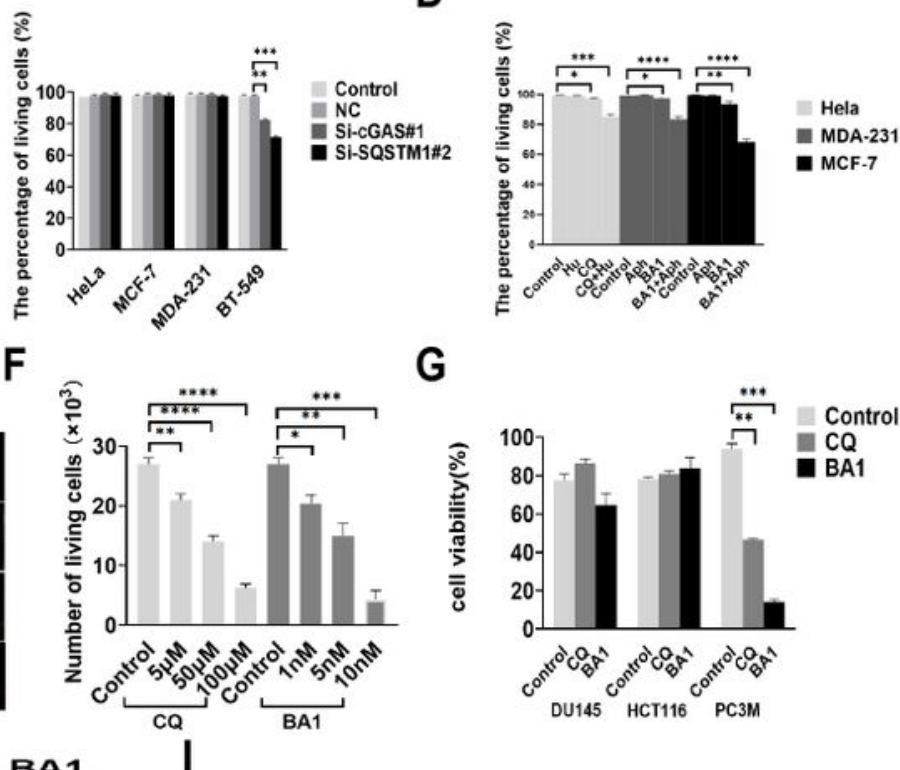

G

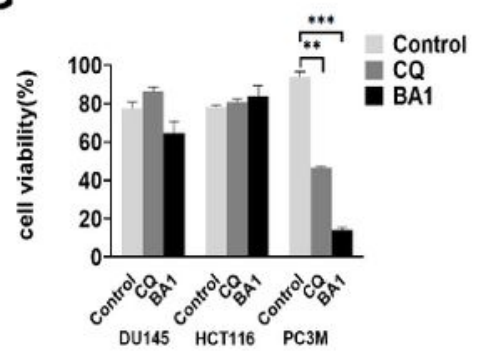

Figure 5

Inhibition of DNA autophagy induced growth arrest or cell death of cancer cells. a, b The effects of autophagic inhibition on the survival and growth of breast cancer cells. Left: MDA-231 and BT-549 cells were treated with the indicated concentrations of BA1 (bafilomycin A1) or CQ for 36 hours, and cell viability was measured. Right: The growth of the MDA-231 and BT-549 cells was analyzed in the presence of $10 \mathrm{nM}$ BA1 or $50 \mu \mathrm{M}$ CQ for the indicated days. Cell viability was measured by Trypan blue exclusion 
assays. c The effects of downregulation of either CGAS or SQSTM1 on the survival of cancer cells. The MDA-231, BT-549, MCF-7 and HeLa cells were transfected with si-cGAS and si-SQSTM1 for 48 hours, and the post-transfection cell viability was analyzed by live and dead assays. $d$ Enhancement of DNA damage sensitized cancer cells to autophagic inhibitors. The MCF-7, MDA-231, and HeLa cells were treated with hydroxyurea $(0.5 \mathrm{mM})$ or aphidicolin $(1 \mu \mathrm{M})$ for 4 hours and then further incubated in the presence of CQ $(50 \mu \mathrm{M})$ or bafilomycin $\mathrm{A} 1(10 \mathrm{nM})$ for 36 hours. Cell viability was analyzed by live and dead assays. $\mathrm{e}$ Double staining of SQSTM1 (green) and cGAS (red) was performed in the 786-0, HCT116, DU145 and PC3M cells. $f$ The 786-0 cells were treated with the indicated concentrations of CQ or BA1 for 36 hours. Cell viability was measured by live and dead assays. $g$ The effects of autophagic inhibition on the survival of cancer cells. The HCT116, DU145 and PC3M cells were treated with CQ $(50 \mu \mathrm{M})$ or bafilomycin A1 (BA1 $10 \mathrm{nM}$ ) for 36 hours, and cell viability was measured by MTS assays. h Detection of the active form of caspase- 3 in the autophagy-inhibited breast cancer cells. The MDA-231 and BT-549 cells were treated with either CQ $(50 \mu \mathrm{M})$ or BA1 (bafilomycin A1, $10 \mathrm{nM})$ for 36 hours, stained for cl-caspase3 by immunofluorescence (upper) and measured by Western blotting (lower). (i) The inhibition of autophagy induced caspase-independent cell death in the BT-549 cells. After treatment with either CQ (50 $\mu \mathrm{M})$ or BA1 (bafilomycin A1, $10 \mathrm{nM}$ ) for 36 hours, the BT-549 cells were incubated in vivo with dihydroethidium (1 mM) for $\mathbf{3 0}$ minutes, and ethidium-stained cells were counted in a total of $\mathbf{5 0 0}$ cells. The images are representative of repeated experiments. The level of statistical significance was $<0.01(* *),<0.001$ (***), and $<0.001(* * *)$, ns: no significance. Each experiment was performed in triplicate, and all experiments were independently repeated at least three times. The images are representative of repeated experiments.

Figure 6

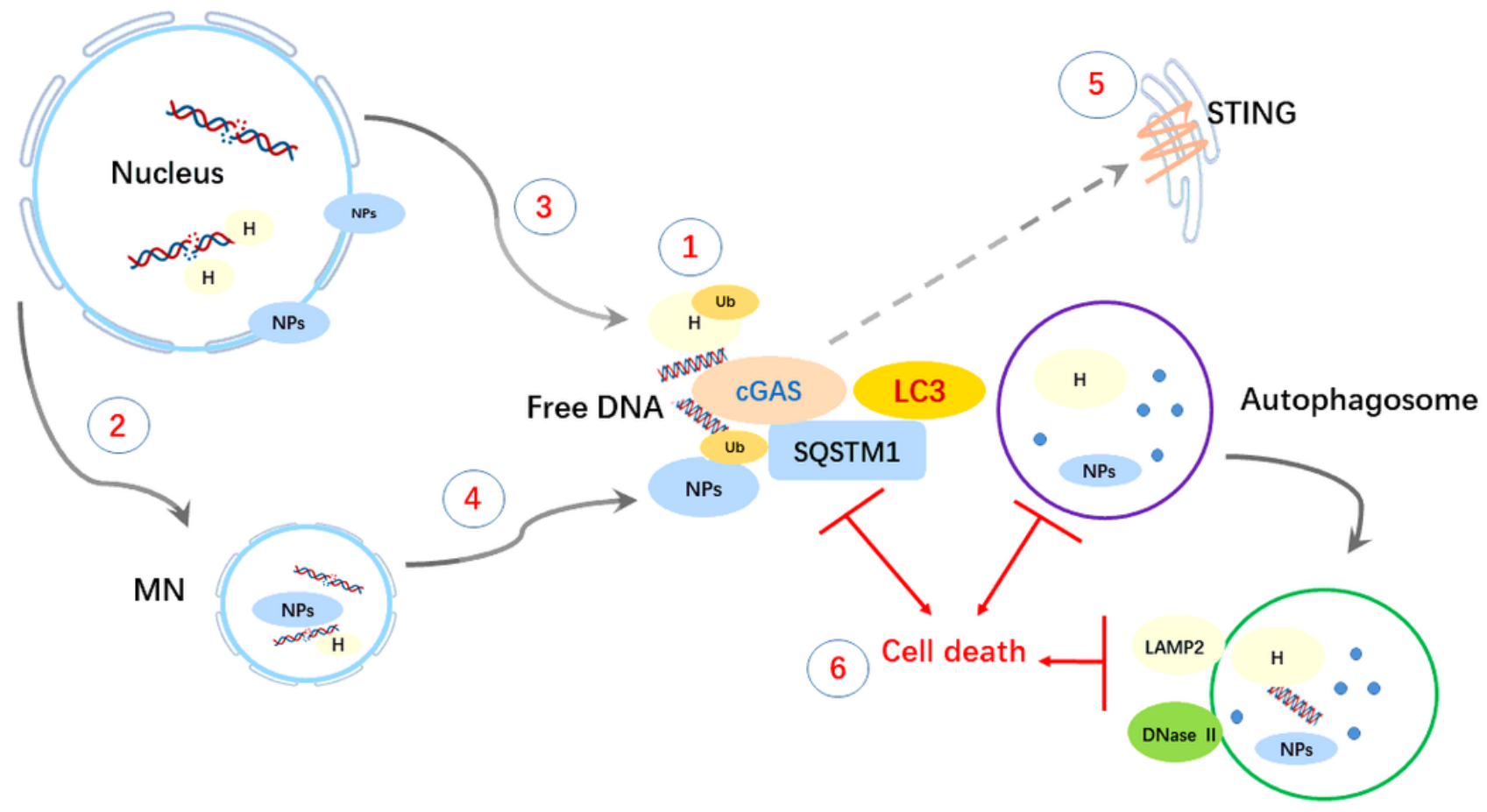

Figure 6 
Schematic summary of the main findings. Cytoplasmic DNA autophagy is initiated by the binding of cGAS to free DNA and may be coordinately accompanied by the recognition of ubiquitinated $(\mathrm{Ub})$ histones $(\mathrm{H})$ or nuclear proteins (NPS) by SQSTM1. 『Cytoplasmic DNA (and proteins) could be derived from either DNA replication-related nuclear damage $\nabla$ and consequent $M N$ formation $\Downarrow$, which would undergo collapse owing to its unscheduled replication $\triangle$. $\otimes$ The degradation of STING could be involved in autophagy and result in a failure to activate SASP activity, but the role of STING in DNA autophagy still needs further exploration. $\nabla$ Generally, in autophagy, free DNA and proteins are enclosed in autophagosomes via LC3 lipidation and degraded after fusion with LAMP2- and DNase II-containing lysosomes. The clearance of cytoplasmic DNA by autophagy could play a protective role in maintaining the growth and survival of cancer cells, and thus, disruption of the process could lead to caspase-independent cell death (CICD).

\section{Supplementary Files}

This is a list of supplementary files associated with this preprint. Click to download.

- SupplementaryFigure.pdf 